\title{
Totally umbilic surfaces in homogeneous 3-manifolds
}

\author{
Rabah Souam and Eric Toubiana
}

\begin{abstract}
We discuss existence and classification of totally umbilic surfaces in the model geometries of Thurston and the Berger spheres. We classify such surfaces in $\mathbb{H}^{2} \times \mathbb{R}, \mathbb{S}^{2} \times \mathbb{R}$ and the Sol group. We prove nonexistence in the Berger spheres and in the remaining model geometries other than the space forms.
\end{abstract}

Mathematics Subject Classification (2000). 53C30, 53B25.

Keywords. Totally umbilic, totally geodesic, homogeneous 3-manifolds.

\section{Introduction}

During the recent years, there has been a rapidly growing interest in the geometry of surfaces in $\mathbb{S}^{2} \times \mathbb{R}$ and $\mathbb{H}^{2} \times \mathbb{R}$ focusing on minimal and constant mean curvature surfaces. This was initiated by H. Rosenberg, [16]. More generally many works are devoted to studying the geometry of surfaces in homogeneous 3-manifolds. See for example [14], [6], [7], [17], [15], [12], [13], [11], [9], [4], [2], [10], [5] and [8].

In the space forms the classification of totally umbilic surfaces is well known and very useful, see [21]. In $\mathbb{R}^{3}$ they are planes and round spheres and in $\mathbb{S}^{3}$ they are round spheres. In $\mathbb{H}^{3}$ they are totally geodesic planes and their equidistants, horospheres and round spheres. In particular they all have constant mean curvature.

A natural question is to understand the totally umbilic surfaces in the remaining homogeneous 3-manifolds. Until now the only known result in this direction was the non-existence of totally umbilic surfaces in the Heisenberg space due to A. Sanini, see [17]. In this paper we study totally umbilic surfaces in simply connected and homogeneous 3-manifolds. More precisely we first consider the manifolds having a 4-dimensional isometry group, denoted by $\mathbb{M}^{3}(\kappa, \tau)$ (see Section 2 ). Namely these manifolds are $\mathbb{S}^{2}(\kappa) \times \mathbb{R}(\kappa>0, \tau=0), \mathbb{H}^{2}(\kappa) \times \mathbb{R}(\kappa<0, \tau=0)$, the Berger spheres $(\kappa>0, \tau \neq 0)$ and the manifolds having the isometry group of either the Heisenberg space $(\kappa=0, \tau \neq 0)$ or $\widehat{\mathrm{PSL}_{2}(\mathbb{R})}(\kappa<0, \tau \neq 0)$, see [3], [19] or [22]. Except for the Berger spheres, these manifolds are four of the eight model geometries of Thurston [22]. The remaining model geometries are the three space forms and the 
Sol geometry which has a 3-dimensional isometry group. As a matter of fact we also consider the Sol geometry.

In Section 2 we prove (Theorem 1) the non-existence of totally umbilic surfaces, in particular the non-existence of totally geodesic ones, in the homogeneous manifolds $\mathbb{M}^{3}(\kappa, \tau)$ for $\tau \neq 0$, that is those which are not Riemannian products. This extends the result of Sanini, [17].

In Section 3 we construct and classify all rotational and totally umbilic surfaces in $\mathbb{S}^{2}(\kappa) \times \mathbb{R}$. In Section 4 we construct and classify all totally umbilic surfaces in $\mathbb{H}^{2}(\kappa) \times \mathbb{R}$ which are invariant under a one-parameter group of ambient isometries. Except for the totally geodesic ones, these surfaces do not have constant mean curvature. In Section 5, we prove that the surfaces obtained in Sections 3 and 4 are the only totally umbilic surfaces in respectively $\mathbb{S}^{2}(\kappa) \times \mathbb{R}$ and $\mathbb{H}^{2}(\kappa) \times \mathbb{R}$.

In Section 6 we show that there exist, up to ambient isometries, only two totally umbilic surfaces in Sol, one of them being totally geodesic.

Finally, in Section 7 we apply our results to prove that any conformal diffeomorphism of $\mathbb{H}^{2} \times \mathbb{R}, \mathbb{S}^{2} \times \mathbb{R}$ and Sol is an isometry.

Throughout this paper all the surfaces are assumed of class $C^{3}$, see however the remark 18.

We are grateful to $\mathrm{H}$. Rosenberg for valuable comments and to the referee for his observations which improved the paper.

\section{Non-existence of totally umbilic surfaces in some homogeneous 3-manifolds}

In this section we consider the connected and simply connected homogeneous Riemannian 3-manifolds, whose isometry group has dimension 4 and which are not Riemannian products. We recall that such a manifold is a fibration over some complete and simply connected surface, $\mathbb{M}^{2}(\kappa)$, of constant curvature $\kappa \in \mathbb{R}$, with geodesic fibers. Actually, for each $\kappa$, there is a one-parameter family $\mathbb{M}^{3}(\kappa, \tau)$ of such fibrations, parametrized by the bundle curvature $\tau \in \mathbb{R}^{*}$. The unit vector field $\xi$ tangent to the fibers is a Killing field and satisfies

$$
\bar{\nabla}_{X} \xi=\tau(X \wedge \xi)
$$

for any tangent vector $X$ in $T \mathbb{M}^{3}(\kappa, \tau)$, where $\bar{\nabla}$ is the connection on $\mathbb{M}^{3}(\kappa, \tau)$. The field $\xi$ defines the vertical direction of the Riemannian submersion $\mathbb{M}^{3}(\kappa, \tau) \rightarrow$ $\mathbb{M}^{2}(\kappa)$. As a matter of fact, the bundle curvature $\tau$ can be zero, but in this case $\mathbb{M}^{3}(\kappa, 0)$ is just a Riemannian product $\mathbb{M}^{2}(\kappa) \times \mathbb{R}$. These product manifolds will be considered in the following sections. Moreover we assume $\kappa-4 \tau^{2} \neq 0$, otherwise the manifold is a space form and its isometry group has dimension 6 . These manifolds are of three types: when $\kappa>0$ they are the Berger spheres, for $\kappa=0$ they have the 
isometry group of the Heisenberg space, $\mathrm{Nil}_{3}$, and for $\kappa<0$ they have the isometry group of $\overline{\mathrm{PSL}_{2}(\mathbb{R})}$.

For more details we refer to [3], [19] and [22].

We can now state our result.

Theorem 1. There exist no totally umbilic surfaces (even non complete) in the 3manifolds $\mathbb{M}^{3}(\kappa, \tau)$, with $\tau \neq 0$ and $\kappa-4 \tau^{2} \neq 0$. In particular, there are no totally geodesic surfaces.

For the special case of the Heisenberg space $(\kappa=0, \tau=1 / 2)$, we recover the result proved by A. Sanini, see [17].

Proof. Let $S$ be an immersed totally umbilic surface in $\mathbb{M}^{3}(\kappa, \tau)$. Locally $S$ is the image of an embedding $X: \Omega \rightarrow \mathbb{M}^{3}(\kappa, \tau)$, where $\Omega$ is an open disk in $\mathbb{R}^{2}$. Call $(u, v)$ the coordinates on $\Omega$ and consider a unit normal field $N$ on $X(\Omega)$. As $X$ is totally umbilic, there exists a function $\lambda: \Omega \rightarrow \mathbb{R}$ such that

$$
\begin{aligned}
& \bar{\nabla}_{X_{u}} N=\lambda X_{u}, \\
& \bar{\nabla}_{X_{v}} N=\lambda X_{v} .
\end{aligned}
$$

Therefore

$$
\begin{aligned}
& \bar{\nabla}_{X_{v}}\left(\bar{\nabla}_{X_{u}} N\right)=\lambda_{v} X_{u}+\lambda \bar{\nabla}_{X_{v}} X_{u}, \\
& \bar{\nabla}_{X_{u}}\left(\bar{\nabla}_{X_{v}} N\right)=\lambda_{u} X_{v}+\lambda \bar{\nabla}_{X_{u}} X_{v} .
\end{aligned}
$$

Subtracting the second equation from the first one we get

$$
\bar{\nabla}_{X_{v}}\left(\bar{\nabla}_{X_{u}} N\right)-\bar{\nabla}_{X_{u}}\left(\bar{\nabla}_{X_{v}} N\right)=\lambda_{v} X_{u}-\lambda_{u} X_{v}
$$

that is,

$$
R\left(X_{u}, X_{v}\right) N=\lambda_{v} X_{u}-\lambda_{u} X_{v},
$$

where $R$ denotes the curvature tensor of $\mathbb{M}^{3}(\kappa, \tau)$.

We define the function $v$ on $\Omega$ setting $v:=\langle N, \xi\rangle$. We denote by $T$ the projection of $\xi$ on $S$, that is $T=\xi-v N$.

As the projection $\mathbb{M}^{3}(\kappa, \tau) \rightarrow \mathbb{M}^{2}(\kappa)$ is a Riemannian submersion, we have the following formula derived by Daniel, see [7]:

$$
R\left(X_{u}, X_{v}\right) N=\left(\kappa-4 \tau^{2}\right) v\left(\left\langle X_{v}, T\right\rangle X_{u}-\left\langle X_{u}, T\right\rangle X_{v}\right) .
$$

Taking into account the relation (2) we get

$$
\nabla \lambda=\left(\kappa-4 \tau^{2}\right) \nu T,
$$

where $\nabla$ denotes the gradient on $S$. 
Observe that if $T=0$ on a nonempty open set, then we can take $N=\xi$ on this set and we deduce from (1) that this surface cannot be umbilic. We can thus assume that $T$ does not vanish on $\Omega$.

Set $J T=N \wedge T$, thus $J T$ is tangent to $S$ and horizontal.

Claim. We have $[T, J T] \equiv 0$.

We need to show that $\bar{\nabla}_{T} J T=\bar{\nabla}_{J T} T$. Since $J T=N \wedge T=N \wedge(\xi-v N)=$ $N \wedge \xi$, we have using (1)

$$
\bar{\nabla}_{T} J T=\bar{\nabla}_{T}(N \wedge \xi)=\bar{\nabla}_{T} N \wedge \xi+N \wedge \bar{\nabla}_{T} \xi=\lambda(T \wedge \xi)+\tau(N \wedge(T \wedge \xi)) .
$$

As $T \wedge \xi=T \wedge(T+v N)=v(T \wedge N)=-v J T$, we deduce that

$$
\bar{\nabla}_{T} J T=-\lambda \nu J T+\tau \nu T .
$$

On the other hand,

$$
\bar{\nabla}_{J T} T=\bar{\nabla}_{J T}(\xi-v N)=\tau(J T \wedge \xi)-J T .(v) N-\lambda v J T .
$$

We have

$$
J T \wedge \xi=J T \wedge(T+v N)=J T \wedge T+v J T \wedge N=-|T|^{2} N+v T,
$$

and

$$
\begin{aligned}
J T .(v) & =J T .\langle N, \xi\rangle=\left\langle\bar{\nabla}_{J T} N, \xi\right\rangle+\left\langle N, \bar{\nabla}_{J T} \xi\right\rangle \\
& =\lambda\langle J T, \xi\rangle+\tau\langle N, J T \wedge \xi\rangle=\tau\langle N, J T \wedge \xi\rangle .
\end{aligned}
$$

Using (5) we obtain

$$
J T .(v)=-\tau|T|^{2} .
$$

Inserting (5) and (6) in (4) we end with

$$
\bar{\nabla}_{J T} T=\tau \nu T-\lambda \nu J T=\bar{\nabla}_{T} J T,
$$

which proves the claim.

Now (3) implies

$$
\begin{aligned}
J T .(\lambda) & =0, \\
T .(\lambda) & =\left(\kappa-4 \tau^{2}\right) \nu|T|^{2}=\left(\kappa-4 \tau^{2}\right) v\left(1-v^{2}\right)
\end{aligned}
$$

Since $[T, J T]=0$, we get $\left(\kappa-4 \tau^{2}\right) J T$. $\left(v-v^{3}\right)=0$. As $\kappa-4 \tau^{2} \neq 0$ we infer that $\left(1-3 v^{2}\right) J T .(v)=0$. This implies easily

$$
J T .(v)=0 .
$$

As JT.(v) $=-\tau|T|^{2}$, see (6), and $\tau \neq 0$, we deduce that $T \equiv 0$, which is a contradiction. This concludes the proof. 


\section{Symmetric totally umbilic surfaces in $\mathbb{S}^{2}(\kappa) \times \mathbb{R}$}

In this section we classify totally umbilic surfaces which are rotationally invariant in $\mathbb{S}^{2} \times \mathbb{R}$. The classification in $\mathbb{M}^{2}(\kappa) \times \mathbb{R}$, for any $\kappa>0$ is completely analogous. We will see that besides the obvious totally geodesic ones, up to isometries of $\mathbb{S}^{2} \times \mathbb{R}$, there are two one-parameter families of complete totally umbilic rotationally invariant surfaces homeomorphic to the two-sphere and a unique complete surface which has the topology of $\mathbb{R}^{2}$. The surfaces of the first family are homologous to zero and those of the second family are not. Moreover these surfaces are embedded, analytic and any totally umbilic rotationally invariant surface is a part of one of these complete surfaces.

A rotational surface in $\mathbb{S}^{2} \times \mathbb{R}$ is by definition a surface obtained by rotating a curve in a totally geodesic cylinder $\mathcal{C}:=\Gamma \times \mathbb{R}$, where $\Gamma \subset \mathbb{S}^{2}$ is a geodesic, around an axis $R=\{p\} \times \mathbb{R}$ where $p$ is a fixed point of $\Gamma$.

In the coordinates $(x, y, t)$ given by the stereographic projection with respect to the north pole, the metric on $\mathbb{S}^{2} \times \mathbb{R}$ reads as follows:

$$
\mathrm{d} \tilde{s}^{2}=\left(\frac{2}{1+x^{2}+y^{2}}\right)^{2}\left(\mathrm{~d} x^{2}+\mathrm{d} y^{2}\right)+\mathrm{d} t^{2},
$$

where $x, y, t \in \mathbb{R}$.

Up to an ambient isometry we can assume that $\Gamma \subset \mathbb{S}^{2}$ corresponds to the complete geodesic defined by $y=0$ and that $p=(0,0,0) \in \Gamma$ is the south pole of $\mathbb{S}^{2}$. Therefore the axis is $R=\{(0,0, t), t \in \mathbb{R}\}$.

Remark 2. Let us remark that for any given curve $\alpha$ in the cylinder $\mathcal{C}$, the surface generated by rotating $\alpha$ around the axis through the south pole, that is $R$, is the same as the one generated by rotating $\alpha$ around the axis through the north pole.

We consider the vertical (noncomplete) geodesic plane $P=\{y=0\} \subset \mathcal{C}$, that is $C=P \cup(\{N\} \times \mathbb{R})$, where $N \in \mathbb{S}^{2}$ is the north pole.

Let $\rho \in]-\pi, \pi[$ denote the signed distance to the origin $(0,0)$ on $\Gamma \cap P$. Thus we have $x=\tan (\rho / 2)$. In the coordinates $(\rho, t)$ the metric on the plane $P$ writes

$$
\mathrm{d} s^{2}=\mathrm{d} \rho^{2}+\mathrm{d} t^{2} .
$$

Consider now a smooth curve $\alpha(s)=(\rho(s), t(s))$ parametrized by arclength in $P$. Let $\theta(s)$ be the oriented angle between the $\rho$-axis and $\alpha^{\prime}(s)$. Therefore, we have

$$
\begin{aligned}
& \rho^{\prime}(s)=\cos \theta(s), \\
& t^{\prime}(s)=\sin \theta(s) .
\end{aligned}
$$

In the plane $P$ we consider the unit normal $N$ to the curve $\alpha$ so that the basis $\left(\alpha^{\prime}(s), N(s)\right)$ is positively oriented for each $s$. We orient by $N$ the symmetric surface 
generated by $\alpha$. The principal curvatures computed with respect to this orientation are as follows:

$$
\begin{aligned}
& \lambda_{1}(s)=\theta^{\prime}(s), \\
& \lambda_{2}(s)=\frac{\sin \theta(s)}{\tan \rho(s)} .
\end{aligned}
$$

Thus, the umbilicity condition is

$$
\theta^{\prime}(s)=\frac{\sin \theta(s)}{\tan \rho(s)}
$$

A priori the equation (8) makes sense only for $\rho \neq 0$, but as we will see later the surfaces we obtain are regular even at such points.

Differentiating the first equation in (7) and using equation (8) we get

$$
\rho^{\prime \prime}=\frac{\left(\rho^{\prime 2}-1\right)}{\tan \rho} .
$$

Assume that $\rho^{\prime}\left(s_{0}\right)=1$ for some $s_{0}$ where $\rho\left(s_{0}\right) \neq 0$. Note that the function $f(s)=\rho\left(s_{0}\right)+s-s_{0}$ is a solution of (9) with the same initial conditions at $s_{0}$ than $\rho$. Therefore $\rho \equiv f$ and $t^{\prime} \equiv 0$ and the surface is part of a slice $\mathbb{S}^{2} \times\left\{t_{0}\right\}$. The same happens in case where $\rho^{\prime}\left(s_{0}\right)=-1$. Henceforth we will assume that $\rho^{\prime 2}(s) \neq 1$ for all $s$ and (9) is equivalent to

$$
\frac{\rho^{\prime \prime}}{\left(\rho^{\prime 2}-1\right)}=\frac{\cos \rho}{\sin \rho}
$$

Multiplying both sides by $2 \rho^{\prime}$ and integrating we get

$$
\rho^{\prime 2}-1=\lambda \sin ^{2} \rho,
$$

for some nonzero real constant $\lambda$. Since the curve $\alpha$ is parametrized by arclength we must have $\rho^{\prime 2}<1$. Thus $\lambda=-a^{2}$ for some $a>0$.

Conversely, any solution $\rho$ of (9) satisfying $\rho^{\prime 2} \leq 1$ defines a function $\theta(s)$ setting $\cos \theta(s)=\rho^{\prime}(s)$. Consider the function $t$ defined by setting $t^{\prime}(s)=a \sin \rho(s)$ and $t\left(s_{0}\right)=t_{0}$ for some $s_{0}$ in the domain of $\rho$ and some real number $t_{0}$. Then $t$ satisfies the second equation of (7) and therefore the curve $\alpha(s)=(\rho(s), t(s)) \in \mathcal{C}$ generates a rotational totally umbilic surface in $\mathbb{S}^{2} \times \mathbb{R}$.

Let $\rho$ be a solution of (9) satisfying $\rho^{\prime 2}<1$. Observe that equations (7) and (8) show that $\rho^{\prime}$ cannot be identically zero on an open interval unless the generated surface is part of the totally geodesic cylinder $\rho \equiv \pi / 2$. Henceforth we assume that $\rho$ is not this trivial solution and so, up to restricting the domain of $\rho$, we can suppose that $\rho$ takes its values in $] 0, \pi / 2[$ or $] \pi / 2, \pi[$. 
So, we can consider an interval on which $\rho^{\prime}$ never vanishes. Changing $s$ into $-s$ if needed we can suppose that $\rho^{\prime}>0$. Therefore we get

$$
\rho^{\prime}=\sqrt{1-a^{2} \sin ^{2} \rho}
$$

It is interesting to note that, when $a \neq 1$, the function $\rho$ is the Jacobi amplitude function $\rho(s)=\operatorname{am}\left(s, a^{2}\right)$ and, up to the sign, we have $t^{\prime}(s)=a \operatorname{sn}\left(s, a^{2}\right)$ and $\theta^{\prime}(s)=a \operatorname{cn}\left(s, a^{2}\right)$, where $\operatorname{sn}\left(s, a^{2}\right)=\sin \operatorname{am}\left(s, a^{2}\right)$ and $\operatorname{cn}\left(s, a^{2}\right)=\cos \operatorname{am}\left(s, a^{2}\right)$ are respectively the sinus and cosinus amplitudinis elliptic Jacobi functions, see for instance [1, Chapter 16] and [18, pp. 286-307]. However, for the reader's convenience and to be self-contained, we will treat in a direct and elementary way this ODE.

Now observe that the transformation $(\rho, t) \mapsto(\pi-\rho, t)$ is an isometry which changes rotations around the axis through the south pole into rotations around the axis through the north pole. Therefore, taking into account Remark 2, we can assume that, up to an ambient isometry, $\rho$ takes its values in $] 0, \pi / 2[$.

Let us call $\rho_{a}$ the maximal solution of (10) extending $\rho$ without restrictions on its values, that is for the moment we do not require that $\rho_{a}$ takes its values in $]-\pi, \pi[$.

Lemma 3. Up to a reparametrization of the form $s \rightarrow s+c$ for some real constant $c$, the maximal solution $\rho_{a}$ is defined on an interval $]-\delta, \delta[$, where $\left.\delta \in] 0,+\infty\right]$. Furthermore $\rho_{a}$ is odd and so satisfies $\rho_{a}(0)=0$ and $\rho_{a}^{\prime}(0)=1$.

Proof. Let us call $] u, v\left[\right.$ the domain of $\rho_{a}$ where $-\infty \leq u<v \leq+\infty$.

We first show that $\rho_{a}$ vanishes at some point. Since $\rho_{a}^{\prime} \leq 1$ such a point clearly exists if $u=-\infty$. In case $u$ is finite, $\rho_{a}$ has a limit $l \in[-\infty,+\infty[$ at $u$ as it is nondecreasing.

If $l<0$ then $\rho_{a}$ vanishes at some point since $\rho$ is positive.

Consider now the case where $l \geq 0$ and call $I \subset] u, v$ [ the domain of $\rho$. Suppose that, as $s$ decreases starting from $I, \rho_{a}$ never vanishes. Then the function $\rho_{a}^{\prime}$ increases (since $\rho(I) \subset] 0, \pi / 2[$ ) and thus has a positive limit at $u$. But then we could extend the solution $\rho_{a}$ of equation (10) beyond $u$, which contradicts the maximality of $\rho_{a}$.

Therefore $\rho_{a}\left(s_{0}\right)=0$ for some point $s_{0}$. Changing $s$ into $s-s_{0}$ we can assume that $\rho_{a}(0)=0$. The function $f(s):=-\rho_{a}(-s)$ is then also a solution of (10) satisfying $f(0)=0$. We conclude that $f \equiv \rho_{a}$, which means $\rho_{a}$ is odd.

Lemma 4. Suppose $a \in] 0,1\left[\right.$. Then $\rho_{a}$ is defined on the whole of $\mathbb{R}$ and it gives rise to a unique, up to an ambient isometry, curve $\alpha_{a}$ generating a rotational totally umbilic surface. This curve is an analytic Jordan curve in the cylinder $\mathcal{C}$, it is nonhomologous to zero and symmetric with respect to the axis of rotation $R$. The rotational totally umbilic surface, $S_{1}(a)$, generated by $\alpha_{a}$ is analytic, embedded and homeomorphic to the sphere. Moreover $S_{1}(a)$ is nonhomologous to zero in $\mathbb{S}^{2} \times \mathbb{R}$. 
The generating curve for $a=0.9$

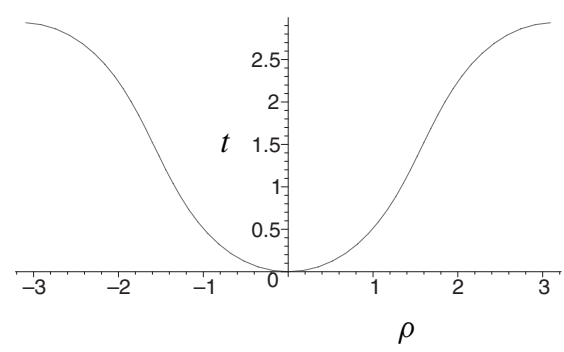

The generating curve for $a=0.5$

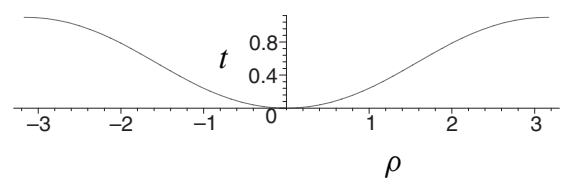

Proof. With the notations of Lemma 3, if $\delta<+\infty$ then, since $\rho_{a}^{\prime}$ takes its values in ]0,1], $\rho_{a}$ would have a finite limit $l$ at $\delta$. Since $\left.a \in\right] 0,1$, we have $1-a^{2} \sin ^{2} l>0$ which allows to extend $\rho_{a}$ beyond $\delta$, contradicting the maximality of $\rho_{a}$. This shows that $\delta=+\infty$.

Since $\rho_{a}^{\prime} \geq \sqrt{1-a^{2}}>0$ and $\rho_{a}(0)=0$, there is a smallest $s_{1}>0$ such that $\rho_{a}\left(s_{1}\right)=\pi$.

Now let us consider the function $f(s):=2 \pi-\rho_{a}\left(2 s_{1}-s\right), s \in \mathbb{R}$. We observe that $f$ is also a solution of equation (10) and satisfies $f\left(s_{1}\right)=2 \pi-\rho_{a}\left(s_{1}\right)=\pi=\rho_{a}\left(s_{1}\right)$. Consequently $f \equiv \rho_{a}$, that is,

$$
\rho_{a}\left(2 s_{1}-s\right)=2 \pi-\rho_{a}(s) \text { for all } s \in \mathbb{R} .
$$

As we are interested in curves generating rotational totally umbilic surfaces, we look for a function $t$ satisfying $t^{\prime 2}=1-\rho_{a}^{\prime 2}=a^{2} \sin ^{2} \rho_{a}$. Let $t_{a}$ be the function defined on $\mathbb{R}$ by setting $t_{a}^{\prime}(s)=a \sin \rho_{a}(s)$ and $t_{a}(0)=0$.

Since $\rho_{a}$ is an odd function and $t_{a}(0)=0$ we deduce that $t_{a}$ is an even function. Observe that the function $g(s):=t_{a}\left(2 s_{1}-s\right)$ satisfies $g^{\prime}(s)=t_{a}^{\prime}(s)$ (using equation (11)) and $g\left(s_{1}\right)=t_{a}\left(s_{1}\right)$. Thus $g \equiv t_{a}$, that is $t_{a}\left(2 s_{1}-s\right)=t_{a}(s)$ for any $s \in \mathbb{R}$. Using the evenness of $t_{a}$ we get

$$
t_{a}\left(s+2 s_{1}\right)=t_{a}(s) \quad \text { for all } s \in \mathbb{R} .
$$

Using equation (11) and the oddness of $\rho_{a}$ we obtain for any $s \in \mathbb{R}$

$$
\rho_{a}(s)=-\rho_{a}(-s)=-\left(2 \pi-\rho_{a}\left(2 s_{1}+s\right)\right),
$$

and so

$$
\rho_{a}\left(s+2 s_{1}\right)=2 \pi+\rho_{a}(s) \text { for all } s \in \mathbb{R} .
$$

Now the curve $\tilde{\alpha}_{a}(s)=\left(\rho_{a}(s), t_{a}(s)\right), s \in \mathbb{R}$, is a curve in the Riemannian universal cover $\tilde{\mathcal{E}}$ of $\mathcal{C}$. Observe that the equations (12) and (13) show that restricting $s$ to $\left[-s_{1}, s_{1}\right], \tilde{\alpha}_{a}$ gives rise to an analytic closed curve $\alpha_{a}$ in $\mathcal{C}$. Since $\rho_{a}$ is increasing 
on $\left[-s_{1}, s_{1}\right]$ and $\rho_{a}\left(-s_{1}\right)=-\rho_{a}\left(s_{1}\right)=-\pi$ we deduce that $\alpha_{a}$ is embedded and nonhomologous to zero.

As $\rho_{a}$ is odd and $t_{a}$ is even, the curve $\alpha_{a}$ has the desired symmetry.

It is clear that the other choice $t_{a}^{\prime}(s)=-a \sin \rho_{a}(s)$ leads to the curve deduced from $\alpha_{a}$ by the isometry $(\rho, t) \mapsto(\rho,-t)$.

Remark 5. We observe that the curve $\alpha_{a}$ is globally invariant under the isometry $(\rho, t) \mapsto\left(\pi-\rho \bmod (2 \pi), t_{a}\left(2 s_{0}\right)-t\right)$.

Lemma 6. Assume $a=1$. Then $\rho_{1}(s)=\pi / 2-2 \arctan e^{-s}, s \in \mathbb{R}$. This gives rise to a unique, up to an ambient isometry, curve $\alpha_{1}$ in $P \subset \mathcal{C}$ generating a rotational totally umbilic surface. The curve $\alpha_{1}$ is complete, open, embedded and symmetric with respect to the axis of rotation $R$. The rotational totally umbilic surface, $S_{1}$, generated by $\alpha_{1}$ in $\mathbb{S}^{2} \times \mathbb{R}$ is complete, properly embedded, analytic and homeomorphic to $\mathbb{R}^{2}$.

Proof. Since $\rho_{1}$ is the maximal solution of $\rho^{\prime}=\sqrt{1-\sin ^{2} \rho}$ satisfying $\rho(0)=0$, we deduce that $\rho_{1}$ is solution of $\rho^{\prime}=\cos \rho$. A straightforward computation shows that the maximal solution of this last equation with the initial condition $\rho(0)=0$ is

$$
\rho_{1}(s)=\frac{\pi}{2}-2 \arctan e^{-s} \quad \text { for all } s \in \mathbb{R} .
$$

Note that $\rho_{1}$ takes its values in $]-\pi / 2, \pi / 2[$.

As in the proof of Lemma 4, we can assume that $t$ satisfies $t^{\prime}(s)=\sin \rho_{1}(s)$, up to an ambient isometry. We consider the function $t_{1}$ defined by setting $t_{1}^{\prime}(s)=\sin \rho_{1}(s)$ and $t_{1}(0)=0$. It is straightforward to check that $\sin \rho_{1}(s)=\tanh (s)$ and then $t_{1}(s)=\log \cosh (s)$.

As $\rho_{1}$ is odd and $t_{1}$ is even, the curve $\alpha_{1}$ has the desired symmetry. As a matter of fact, $\alpha_{1}$ is the graph of the function $\left.t(\rho)=-\log \cos \rho, \rho \in\right]-\pi / 2, \pi / 2[$. This concludes the proof.

Lemma 7. Assume $a>1$. Then the maximal solution $\rho_{a}$ is defined on a bounded interval $]-\delta_{a}, \delta_{a}\left[\right.$ where $\delta_{a}$ is a positive number. It gives rise to a unique, up to an ambient isometry, Jordan curve $\alpha_{a}$ in $P \subset \mathcal{C}$ generating a rotational totally umbilic surface. The curve $\alpha_{a}$ is analytic and symmetric with respect to the axis of rotation $R$. The rotational totally umbilic surface, $S_{2}(a)$, generated by $\alpha_{a}$ is analytic, embedded and homeomorphic to the sphere. Furthermore, $S_{2}(a)$ is homologous to zero in $\mathbb{S}^{2} \times \mathbb{R}$.

Proof. Since $a>1$ we deduce that $\left.\rho_{a}(s) \in\right]-\arcsin 1 / a$, $\arcsin 1 / a[$. Recall that $\rho_{a}$ is defined on an open interval ] $-\delta_{a}, \delta_{a}$ [, see Lemma 3. We first show that $\delta_{a}$ is finite. Assume by contradiction $\delta_{a}=+\infty$. Since $\rho_{a}$ is nondecreasing it admits a 
The generating curve for $a=1$

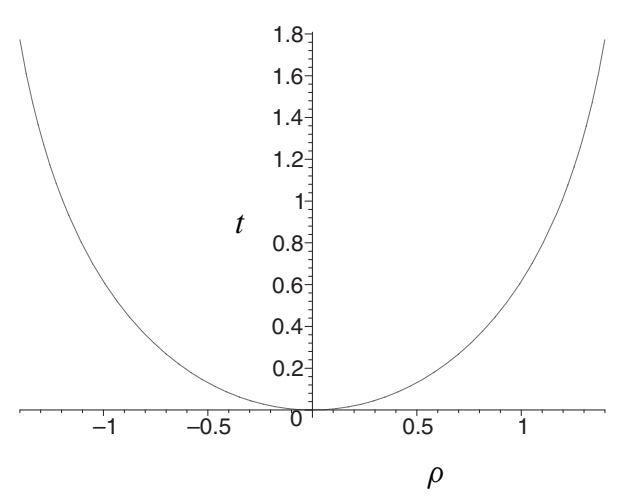

limit $l \in] 0, \arcsin 1 / a]$ as $s \rightarrow+\infty$. Necessarily $l=\arcsin 1 / a$ since otherwise it would follow from the equation (10) that $\rho_{a}^{\prime}>\sqrt{1-a^{2} \sin ^{2} l}>0$ for all $s>0$. Therefore $\rho_{a}$ would not be bounded.

Using the equation (9) we see that for $s$ big enough, $\rho_{a}^{\prime \prime}(s) \leq-1 /(2 \tan l)<0$. Consequently $\rho_{a}^{\prime}$ would be negative for $s$ big enough which is a contradiction. This proves that $\delta_{a}$ is finite.

Let us call again $l$ the limit of $\rho_{a}$ as $s \rightarrow \delta_{a}$. If $l<\arcsin 1 / a$ then we could extend the solution $\rho_{a}$, which is maximal, beyond $\delta_{a}$, which is absurd. So $l=\arcsin 1 / a$ and $\rho_{a}^{\prime} \rightarrow 0$ as $s \rightarrow \delta_{a}$.

Observe that, since the function $\rho_{a}$ satisfies equations (9) and (10), it satisfies also the following equation

$$
\rho^{\prime \prime}=-a^{2} \sin \rho \cos \rho .
$$

As the second member of (14) is bounded, its maximal solutions are defined on the whole of $\mathbb{R}$. Call $\tilde{\rho}_{a}$ the maximal solution of (14) extending $\rho_{a}$. Set $f(s):=$ $\tilde{\rho}_{a}\left(2 \delta_{a}-s\right)$. It is clear that $f$ and $\tilde{\rho}_{a}$ satisfy equation (14) with the same initial conditions at $\delta_{a}$. Thus we have

$$
\tilde{\rho}_{a}\left(2 \delta_{a}-s\right)=\tilde{\rho}_{a}(s) \quad \text { for all } s \in \mathbb{R} .
$$

As we are interested in curves generating rotational totally umbilic surfaces, we look for a function $t$ satisfying $t^{\prime 2}=1-\tilde{\rho}_{a}^{\prime 2}=a^{2} \sin ^{2} \tilde{\rho}_{a}$. Let $t_{a}$ be the function defined by $t_{a}^{\prime}=a \sin \tilde{\rho}_{a}$ and $t_{a}(0)=0$. As $\tilde{\rho}_{a}$ is an odd function we deduce that $t_{a}$ is even. Observe that the function $g(s):=2 t_{a}\left(\delta_{a}\right)-t_{a}\left(2 \delta_{a}-s\right)$ satisfies $g^{\prime}(s)=t_{a}^{\prime}(s)$ (using equation (15)) and $g\left(\delta_{a}\right)=t_{a}\left(\delta_{a}\right)$. Thus $g \equiv t_{a}$, that is

$$
t_{a}\left(2 \delta_{a}-s\right)=2 t_{a}\left(\delta_{a}\right)-t_{a}(s) \quad \text { for all } s \in \mathbb{R} .
$$


It follows from (15) and the oddness of $\tilde{\rho}_{a}$ that

$$
\tilde{\rho}_{a}\left(s+4 \delta_{a}\right)=\tilde{\rho}_{a}(s) \quad \text { for all } s \in \mathbb{R} .
$$

In the same way, using (16) and the evenness of $t_{a}$, we get

$$
t_{a}\left(s+4 \delta_{a}\right)=t_{a}(s) \quad \text { for all } s \in \mathbb{R} .
$$

Now the curve $\alpha_{a}(s)=\left(\tilde{\rho}_{a}(s), t_{a}(s)\right), s \in \mathbb{R}$, parametrizes a closed analytic curve in $P \subset \mathcal{C}$. Taking into account (15), the oddness of $\tilde{\rho}_{a},(16)$ and the evenness of $t_{a}$, we deduce that the curve $\alpha_{a}$ is symmetric with respect to the axis $R$.

Considering the fact that $t_{a}$ is increasing on $\left[0,2 \delta_{a}\right]$ and the symmetry of $\alpha_{a}$, we infer that $\alpha_{a}$ defines a Jordan curve in $P$.

To conclude the proof observe that the other choice for $t_{a}$, that is $t_{a}^{\prime}=-a \sin \tilde{\rho}_{a}$, leads to the curve deduced from $\alpha_{a}$ by the isometry $(\rho, t) \mapsto(\rho,-t)$.

The generating curve for $a=1.1$

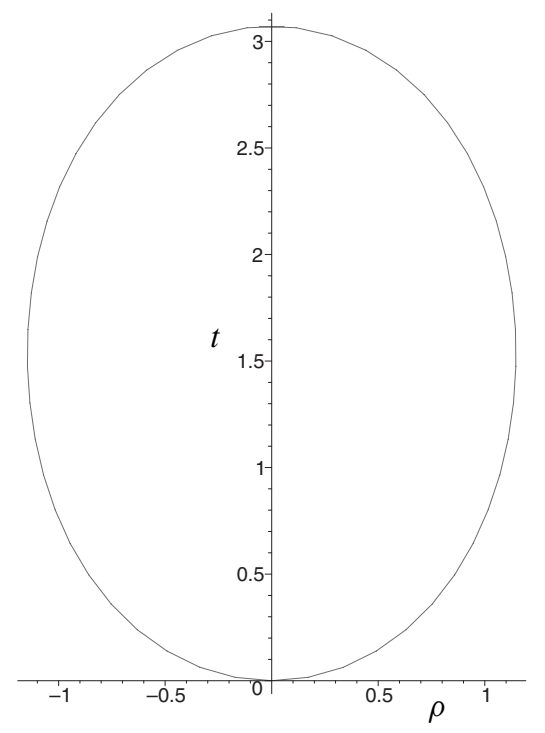

$$
\text { The generating curve for } a=5
$$

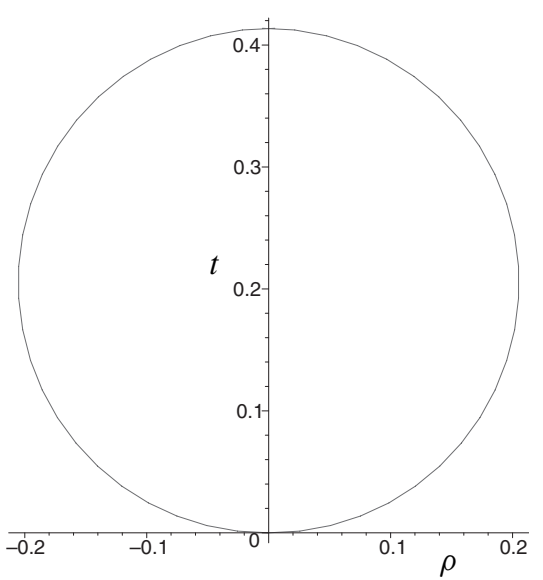

Remark 8. The relations (15) and (16) show that the curve $\alpha_{a}$ in Lemma 7 is symmetric with respect to the horizontal reflection $(\rho, t) \mapsto\left(\rho, 2 t_{a}\left(\delta_{a}\right)-t\right)$ in $P$. Consequently, the surface $S_{3}(a)$ is symmetric with respect to the slice $\mathbb{S}^{2} \times\left\{t_{a}\left(\delta_{a}\right)\right\}$.

Furthermore, we observe that each surface $S_{3}(a)$ is contained in $\mathbb{S}_{-}^{2} \times \mathbb{R}$ where $\mathbb{S}_{-}^{2}$ is the south hemispshere. 
Summarizing we can state the following result.

Theorem 9. Besides the totally geodesic slices $\mathbb{S}^{2} \times\left\{t_{0}\right\}$ and the vertical cylinder $\Gamma \times \mathbb{R}$ where $\Gamma \subset \mathbb{S}^{2}$ is a geodesic, the surfaces introduced in Lemmas 4, 6 and 7 are, up to ambient isometries, the only complete totally umbilic rotational surfaces in $\mathbb{S}^{2} \times \mathbb{R}$. In particular they are all embedded and homeomorphic either to $\mathbb{R}^{2}$ or to $\mathbb{S}^{2}$. Among the surfaces homeomorphic to $\mathbb{S}^{2}$ some are homologous to zero and some are not.

Remark 10. It is interesting to observe that unlike in the case of space forms, the totally umbilic surfaces we obtained do not have constant mean curvature, except for the totally geodesic ones.

\section{Symmetric totally umbilic surfaces in $\mathbb{H}^{2}(\kappa) \times \mathbb{R}$}

In this section, we classify the totally umbilic surfaces in $\mathbb{H}^{2} \times \mathbb{R}$ which are invariant under a one-parameter group of isometries. The case of $\mathbb{M}^{2}(\kappa) \times \mathbb{R}, \kappa<0$, is completely similar.

We recall that in $\mathbb{H}^{2}$ there are three kinds of one-parameter families of positive isometries: the rotations around a fixed point (elliptic isometries), the translations along a fixed geodesic (hyperbolic isometries) and the "translations" along the horocycles sharing the same point at infinity (parabolic isometries). An isometry of $\mathbb{H}^{2}$ obviously induces an isometry of $\mathbb{H}^{2} \times \mathbb{R}$ fixing the factor $\mathbb{R}$ pointwise. Such an isometry of $\mathbb{H}^{2} \times \mathbb{R}$ obtained from an elliptic (resp. parabolic, hyperbolic) isometry of $\mathbb{H}^{2}$ will thus be called elliptic (resp. parabolic, hyperbolic).

We will see that for each of the associated families of isometries of $\mathbb{H}^{2} \times \mathbb{R}$ there exist complete and globally invariant totally umbilic surfaces. In fact, we are going to classify all of them. More precisely, we prove they are all embedded, those which are invariant under elliptic isometries are either totally geodesic slices $\mathbb{H}^{2} \times\left\{t_{0}\right\}$ or homeomorphic to the sphere and the remaining ones are all homeomorphic to the plane. In particular the only totally geodesic ones are the slices and the products $\Gamma \times \mathbb{R}$ where $\Gamma \subset \mathbb{H}^{2}$ is a geodesic.

We will work with the disk model for $\mathbb{H}^{2}$, so that

$$
\mathbb{H}^{2}=\left\{(x, y) \in \mathbb{R}^{2}, x^{2}+y^{2}<1\right\},
$$

and the metric is

$$
\mathrm{d} s_{\mathbb{H}}^{2}=\left(\frac{2}{1-\left(x^{2}+y^{2}\right)}\right)^{2}\left(\mathrm{~d} x^{2}+\mathrm{d} y^{2}\right)
$$


Therefore the product metric on $\mathbb{H}^{2} \times \mathbb{R}$ reads as follows:

$$
\mathrm{d} \tilde{s}^{2}=\left(\frac{2}{1-\left(x^{2}+y^{2}\right)}\right)^{2}\left(\mathrm{~d} x^{2}+\mathrm{d} y^{2}\right)+\mathrm{d} t^{2},
$$

where $(x, y) \in \mathbb{H}^{2}$ and $t \in \mathbb{R}$. We consider the following particular geodesics of $\mathbb{H}^{2}$ :

$$
\begin{aligned}
& \Gamma=\{(x, 0), x \in]-1,1[\} \subset \mathbb{H}^{2}, \\
& L=\{(0, y), y \in]-1,1[\} \subset \mathbb{H}^{2} .
\end{aligned}
$$

Up to ambient isometries, we can assume that the symmetric surfaces are generated by curves in the geodesic plane $P:=\Gamma \times \mathbb{R} \subset \mathbb{H}^{2} \times \mathbb{R}$.

On the geodesic $\Gamma$ we denote by $\rho \in \mathbb{R}$ the signed distance to the origin $(0,0)$, thus $x=\tanh \rho / 2$. Therefore the metric on $P$ is

$$
\mathrm{d} s^{2}=\left(\frac{2}{1-x^{2}}\right)^{2} \mathrm{~d} x^{2}+\mathrm{d} t^{2}=\mathrm{d} \rho^{2}+\mathrm{d} t^{2} .
$$

Given a curve $\alpha(s)=(\rho(s), t(s))$ parametrized by arclength in $P$, we let $\theta(s)$ be the oriented angle between the $\rho$-axis and $\alpha^{\prime}(s)$. Therefore, we have

$$
\begin{aligned}
\rho^{\prime}(s) & =\cos \theta(s), \\
t^{\prime}(s) & =\sin \theta(s) .
\end{aligned}
$$

In the elliptic case, the isometries of $\mathbb{H}^{2} \times \mathbb{R}$ under consideration are the rotations around the vertical axis $R:=\{(0,0)\} \times \mathbb{R}$. In the parabolic case, the isometries are the ones corresponding to the point at infinity $(-1,0) \in \partial_{\infty} \mathbb{H}^{2}$. Finally, the hyperbolic isometries correspond to translations along $L$ in $\mathbb{H}^{2}$.

In the plane $P$ we consider the unit normal $N$ to the curve $\alpha$ so that the basis $\left(\alpha^{\prime}(s), N(s)\right)$ is positively oriented for each $s$. In the three cases we orient by $N$ the symmetric surface generated by $\alpha$. The principal curvatures computed with respect to this orientation are as follows:

$$
\lambda_{1}(s)=\theta^{\prime}(s)
$$

and

$$
\lambda_{2}(s)= \begin{cases}\frac{\sin \theta(s)}{\tanh \rho(s)} & (\text { elliptic case), } \\ \sin \theta(s) & (\text { parabolic case) } \\ \sin \theta(s) \tanh \rho(s) & \text { (hyperbolic case) }\end{cases}
$$


4.1. Elliptic case. The umbilicity condition is

$$
\theta^{\prime}(s)=\frac{\sin \theta(s)}{\tanh \rho(s)}
$$

This case is similar to the case $a>1$ in $\mathbb{S}^{2} \times \mathbb{R}$, so we will omit the details.

Differentiating the first equation in (17) and using the umbilicity condition we get

$$
\rho^{\prime \prime}=\frac{\left(\rho^{\prime 2}-1\right)}{\tanh \rho} .
$$

Discarding the trivial totally geodesic surfaces $\mathbb{H}^{2} \times\left\{t_{0}\right\}$, we can show as in the case of $\mathbb{S}^{2} \times \mathbb{R}$ that $\rho^{\prime 2}(s) \neq 1$ for any $s$ such that $\rho(s) \neq 0$. Therefore we may assume that $\rho^{\prime 2}<1$.

We can state the following.

Proposition 11. Any local solution of (18) satisfying $\rho^{\prime 2}<1$ gives rise to a unique, up to ambient isometries, complete rotational totally umbilic and nongeodesic surface in $\mathbb{H}^{2} \times \mathbb{R}$. Moreover, there exists a one-parameter family of such surfaces and all of them are analytic, embedded and homeomorphic to the sphere.

Besides the totally geodesic slices $\mathbb{H}^{2} \times\{t\}$, these surfaces are the only complete rotational and totally umbilic surfaces in $\mathbb{H}^{2} \times \mathbb{R}$.

Furthermore, any rotational umbilic (including geodesic) surface in $\mathbb{H}^{2} \times \mathbb{R}$ is, up to an ambient isometry, part of one of the above surfaces.

Proof. Let $\rho$ be a local solution of (18). Proceeding as in the case of $\mathbb{S}^{2} \times \mathbb{R}$, we can suppose that $\rho^{\prime}>0$ and so

$$
\rho^{\prime}=\sqrt{1-b^{2} \sinh ^{2} \rho}
$$

for some real number $b>0$. As in the case of $\mathbb{S}^{2} \times \mathbb{R}$, the functions $\rho(s)$ and $t(s)$ are related to the Jacobi elliptic functions as follows: $\rho(s)=-i \operatorname{am}\left(i s,-b^{2}\right)$ and, up to the sign, $t^{\prime}(s)=i b \operatorname{sn}\left(i s,-b^{2}\right)$, see [1, Chapter 16]. Again, we prefer to give direct and elementary arguments.

Let $\rho_{b}$ be the maximal solution of (19) extending $\rho$. As in the proof of Lemma 3, we can prove that $\rho_{b}$ vanishes at some point. Thus, up to a reparametrization we can assume that $\rho_{b}(0)=0$, consequently we prove as in Lemma 3 that $\rho_{b}$ is an odd function. Therefore $\rho_{b}$ is defined on an interval $]-\delta_{b}, \delta_{b}[$. As in Lemma 7, it can be shown that $\delta_{b}$ is a finite positive number, that $\rho_{b}$ has a finite limit $l=\operatorname{argsinh} 1 / b$ at $\delta_{b}$ and that $\rho_{b}^{\prime}(s) \rightarrow 0$ as $s \rightarrow \delta_{b}$.

From equations (18) and (19) we deduce that $\rho_{b}$ satisfies

$$
\rho^{\prime \prime}=-b^{2} \cosh \rho \sinh \rho
$$



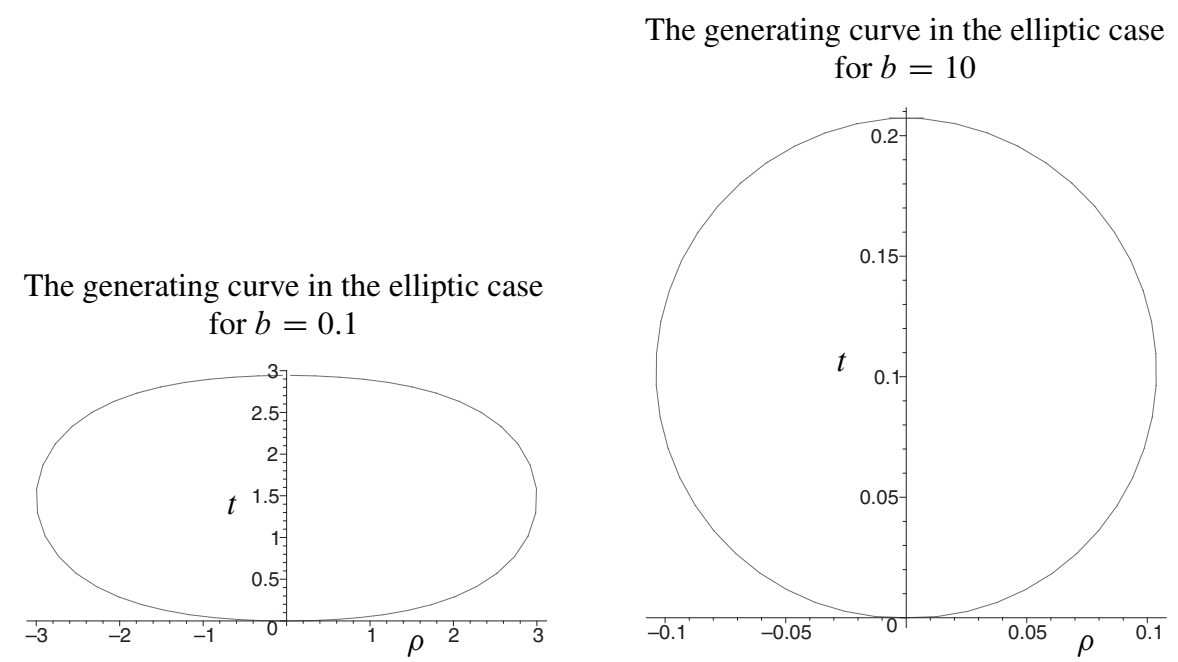

with the initial conditions $\rho(0)=0$ and $\rho^{\prime}(0)=1$. Therefore we can extend the solution $\rho_{b}$ of (20) beyond $\delta_{b}$. Let $\tilde{\rho}_{b}$ be the maximal solution of (20) extending $\rho_{b}$. Observe that for any $s_{0}$ where $\tilde{\rho}_{b}^{\prime}\left(s_{0}\right)=0$ we have the symmetry $\tilde{\rho}_{b}\left(2 s_{0}-s\right)=\tilde{\rho_{b}}(s)$. As $\tilde{\rho}_{b}^{\prime}\left(\delta_{b}\right)=0$ and $\tilde{\rho}_{b}$ is odd, we deduce that $\tilde{\rho}_{b}$ is defined on all of $\mathbb{R}$ and that it is $4 \delta_{b}$-periodic.

As we are interested in curves generating rotational totally umbilic surfaces, we look for a function $t$ satisfying $t^{\prime 2}=1-\tilde{\rho}_{b}^{\prime 2}=b^{2} \sinh ^{2} \tilde{\rho}_{b}$. Let $t_{b}$ be the function defined by $t_{b}^{\prime}=b \sinh \tilde{\rho}_{b}$ and $t_{b}(0)=0$, thus $t_{b}$ is an even function. As in the proof of Lemma 7, we can show that $t_{b}$ satisfies $t_{b}\left(2 \delta_{b}-s\right)=2 t_{b}\left(\delta_{b}\right)-t_{b}(s)$ for any $s \in \mathbb{R}$ and that it is also $4 \delta_{b}$-periodic.

Taking into account that $t_{b}$ is increasing on $\left[0,2 \delta_{b}\right]$, we deduce that the curve $\alpha_{b}(s)=\left(\tilde{\rho}_{b}(s), t(s)\right), s \in \mathbb{R}$, parametrizes an analytic Jordan curve in $P$, symmetric with respect to the axis $R$.

To conclude the proof we just observe that the other choice for $t_{b}$, that is $t_{b}^{\prime}=$ $-b \sinh \tilde{\rho}_{b}$, leads to the curve deduced from $\alpha_{b}$ by the isometry $(\rho, t) \mapsto(\rho,-t)$.

4.2. Parabolic case. The umbilicity condition is

$$
\theta^{\prime}(s)=\sin \theta(s)
$$

Integrating this equation we get

$$
\theta(s)=2 \arctan \lambda e^{s} \quad \text { for all } s \in \mathbb{R},
$$

for some real constant $\lambda$. 
First observe that $\lambda=0$ leads to the curve $\Gamma$ which generates a slice $\mathbb{H}^{2} \times\left\{t_{0}\right\}$.

Now if $\lambda<0$ then $\theta$ is a negative function. Note that the symmetry $(\rho, t) \mapsto$ $(\rho,-t)$ changes $\theta$ into $-\theta$. Therefore, up to an ambient isometry, we can assume that $\theta$ is positive and then $\lambda>0$. Finally observe that up to the reparametrization $s \mapsto s-\log \lambda$ we can assume $\lambda=1$ and then

$$
\theta(s)=2 \arctan e^{s} \quad \text { for all } s \in \mathbb{R} .
$$

Taking into account the first equation in (17) and (21) we obtain

$$
\rho^{\prime}(s)=\cos 2 \arctan e^{s}=-\tanh s \quad \text { for all } s \in \mathbb{R} .
$$

Thus

$$
\rho(s)=-\log \cosh s+\mu \quad \text { for all } s \in \mathbb{R},
$$

for some real constant $\mu$. Note that the isometries of $\mathbb{H}^{2} \times \mathbb{R}$ obtained from the hyperbolic translations along the geodesic $\Gamma$ in $\mathbb{H}^{2}$ send any surface invariant under the parabolic isometries fixing the point at infinity $(-1,0) \in \partial_{\infty} \mathbb{H}^{2}$ to a surface of the same type. Consequently, up to an ambient isometry, we can assume $\mu=0$. Thus

$$
\rho(s)=-\log \cosh s \quad \text { for all } s \in \mathbb{R} .
$$

As for the function $t$, taking into account the second equation in (17) and (21) we obtain

$$
t^{\prime}(s)=\sin 2 \arctan e^{s}=\frac{2 e^{s}}{1+e^{2 s}} \quad \text { for all } s \in \mathbb{R} .
$$

Integrating we get

$$
t(s)=2 \arctan e^{s}+\beta \quad \text { for all } s \in \mathbb{R},
$$

for some real constant $\beta$. Up to a vertical translation we can take $\beta=-\pi / 2$ so that $t(0)=0$ and

$$
t(s)=2 \arctan e^{s}-\frac{\pi}{2} \quad \text { for all } s \in \mathbb{R} .
$$

Note that $t(-s)=-t(s)$ and $\rho(-s)=\rho(s)$ for any $s \in \mathbb{R}$ so that the curve $L_{\text {par }}$ parametrized by $(\rho(s), t(s)), s \in \mathbb{R}$, is symmetric with respect to $\Gamma$.

Summarizing we state the following proposition.

Proposition 12. Besides the slices $\mathbb{H}^{2} \times\{t\}$, up to ambient isometries, there exists a unique complete totally umbilic surface $S_{P}$ in $\mathbb{H}^{2} \times \mathbb{R}$ invariant under parabolic isometries. This surface is analytic, properly embedded, homeomorphic to a plane and is invariant under reflection with respect to a horizontal slice.

Moreover, any totally umbilic surface invariant under parabolic isometries is either part of a slice or, up to an ambient isometry, part of this surface. 
The generating curve in the parabolic case

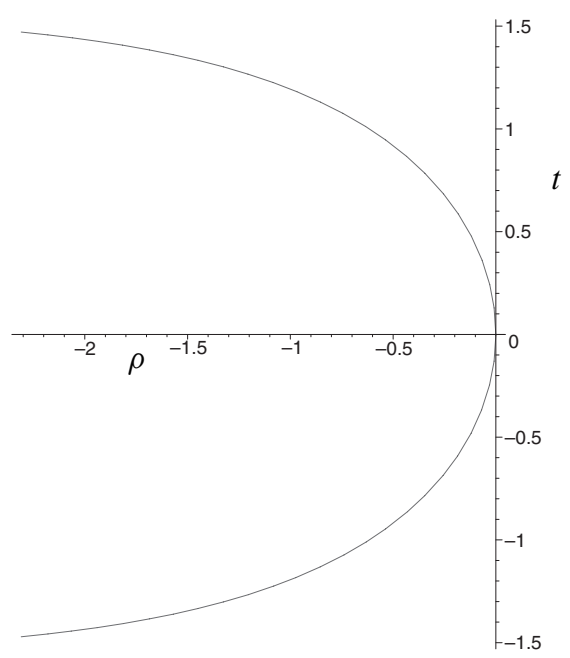

Remark 13. Consider the surface $M$ in $\mathbb{H}^{2} \times \mathbb{R}$ generated by the same curve $L_{\text {par }} \subset P$ under parabolic isometries fixing, now, the point at infinity $(1,0) \in \partial_{\infty} \mathbb{H}^{2}$ (and not $(-1,0)$ as before). Observe that at each point of $M$ the principal curvatures $\tilde{\lambda}_{1}, \tilde{\lambda}_{2}$ of $M$ are given by $\tilde{\lambda}_{1}=\lambda_{1}$ and $\tilde{\lambda}_{2}=-\lambda_{2}$. Therefore we get

$$
\tilde{\lambda}_{1}+\tilde{\lambda}_{2}=\lambda_{1}-\lambda_{2} \equiv 0 \text {. }
$$

We deduce that $M$ is a complete minimal surface of $\mathbb{H}^{2} \times \mathbb{R}$, embedded and invariant under parabolic isometries. Consequently, $M$ is foliated by horocycles. This minimal surface was considered by B. Daniel [6], R. Sa Earp [8] and L. Hauswirth [12].

4.3. Hyperbolic case. The umbilicity condition is

$$
\theta^{\prime}(s)=\sin \theta(s) \tanh \rho(s) .
$$

Proceeding as in the elliptic case, we discard the totally geodesic surfaces $\mathbb{H}^{2} \times\{t\}$ and therefore we can assume that $\rho$ satisfies $\rho^{\prime 2}<1$ and

$$
\rho^{\prime \prime}=\left(\rho^{\prime 2}-1\right) \tanh \rho, \quad \rho^{\prime 2}-1=-c^{2} \cosh ^{2} \rho,
$$

for some real constant $c \in] 0,1[$. Again, invoking the Jacobi elliptic functions, it can be shown that, up to the sign, we have $\rho(s)=i$ am $\left(i s+K, c^{2}\right)-i \pi / 2$ and $t^{\prime}(s)=$ $c \operatorname{sn}\left(i s+K, c^{2}\right)$ where $K=\int_{0}^{\pi / 2} \frac{d t}{\sqrt{1-c^{2} \sin ^{2} t}}$, see [1, Chapter 16]. Nevertheless, as in the previous cases, we prefer to give direct and elementary arguments. 
We deduce from (22) that

$$
\rho^{\prime \prime}=-c^{2} \cosh \rho \sinh \rho .
$$

If $\rho \equiv 0$, then the generated surface is the vertical totally geodesic plane, that is, $\{(0, y, t),-1<y<1, t \in \mathbb{R}\}$. Discarding this case, we consider only the nontrivial solutions of (23).

Again, as in the elliptic case, it can be shown that any maximal solution of the last equation is defined on the whole of $\mathbb{R}$, is periodic, vanishes at some point and, up to a reparametrization, is odd. We can therefore assume that there exists a unique maximal solution $\rho_{c}$ satisfying $\rho(0)=0$ and $\rho^{\prime}(0)=\sqrt{1-c^{2}}$.

As we are interested in curves generating totally umbilic surfaces, we look for a function $t$ satisfying $t^{\prime 2}=1-\rho_{c}^{\prime 2}=c^{2} \cosh ^{2} \rho_{c}$. Let $t_{c}$ be the function defined by $t_{c}^{\prime}=c \cosh \rho_{c}$ and $t_{c}(0)=0$. The function $t_{c}$ is odd. Moreover consider any $s_{0} \in \mathbb{R}$ such that $\rho_{c}^{\prime}\left(s_{0}\right)=0$, then it can be shown that $\rho_{c}\left(2 s_{0}-s\right)=\rho_{c}(s)$. Set $T(s):=-t_{c}\left(2 s_{0}-s\right)+2 t_{c}\left(s_{0}\right), s \in \mathbb{R}$. We have $T^{\prime} \equiv t_{c}^{\prime}$ and $T\left(s_{0}\right)=t_{c}\left(s_{0}\right)$, therefore $T=t_{c}$. As the function $t_{c}$ is odd, we have $t_{c}\left(s+4 s_{0}\right)=4 t_{c}\left(s_{0}\right)+t_{c}(s)$ for every $s \in \mathbb{R}$. We can deduce that $t_{c}$ is an increasing and nonbounded function on $\mathbb{R}$. This shows that the curve $\alpha_{c}=\left(\rho_{c}, t_{c}\right)$ is properly embedded.

Observe that the other choice $t^{\prime}=-c \cosh \rho_{c}$ changes the curve $\alpha_{c}=\left(\rho_{c}, t_{c}\right)$ into the symmetric curve $\left(\rho_{c},-t_{c}\right)$ with respect to $\Gamma$.

The generating curve in the hyperbolic case

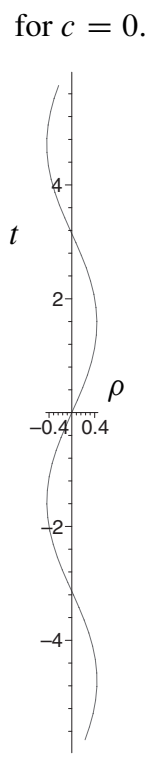

The generating curve in the hyperbolic case for $c=0.1$

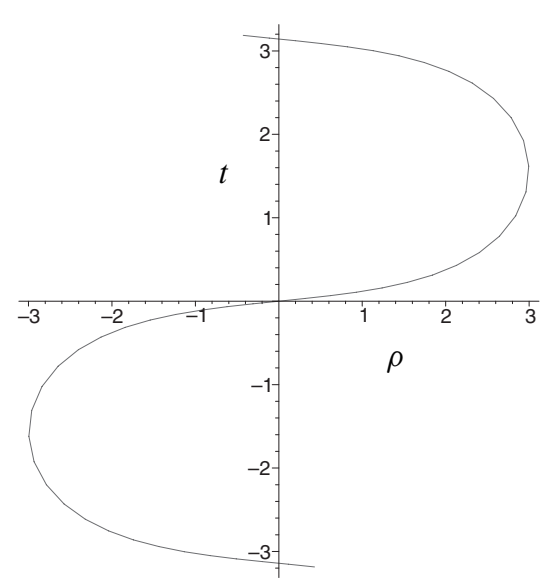


We summarize stating the following.

Proposition 14. Any non identically zero local solution of (23) satisfying $\rho^{\prime 2}<1$ gives rise to a unique, up to ambient isometries, complete totally umbilic and nongeodesic surface $S_{c}, c>0$, in $\mathbb{H}^{2} \times \mathbb{R}$ invariant under hyperbolic isometries. Moreover, there exists a one-parameter family of such surfaces and all of them are analytic, properly embedded and homeomorphic to the plane. These surfaces are periodic in the vertical direction and symmetric with respect to a discrete set of horizontal slices.

Furthermore, any umbilic surface in $\mathbb{H}^{2} \times \mathbb{R}$ invariant under hyperbolic isometries is either part of a vertical totally geodesic plane, a slice $\mathbb{H}^{2} \times\{t\}$ or, up to an ambient isometry, part of one of the surfaces $S_{c}$.

\section{Unicity of totally umbilic surfaces in $\mathbb{H}^{2}(\kappa) \times \mathbb{R}$ and $\mathbb{S}^{2}(\kappa) \times \mathbb{R}$}

In this section $\mathbb{M}^{2}$ stands for $\mathbb{H}^{2}$ or $\mathbb{S}^{2}$. The cases $\mathbb{M}^{2}=\mathbb{M}^{2}(\kappa)$ for $\kappa<0$ and $\kappa>0$ are completely analogous.

We will need the following result which is of independent interest.

Proposition 15. Let $S \subset \mathbb{M}^{2} \times \mathbb{R}$ be an orientable surface transversal to each slice $\mathbb{M}^{2} \times\{t\}$. We suppose the following:

(1) The geodesic curvature of each horizontal curve $S_{t}:=S \cap\left(\mathbb{M}^{2} \times\{t\}\right)$ in $\mathbb{M}^{2}$ is constant (depending on $t$ ).

(2) The angle between $S$ and $\mathbb{M}^{2} \times\{t\}$ is constant along $S_{t}$ for each $t$.

Then:

In case $\mathbb{M}^{2}=\mathbb{S}^{2}$ the surface $S$ is part of rotational surface.

In case $\mathbb{M}^{2}=\mathbb{H}^{2}$ the surface $S$ is part of either

(a) a rotational surface,

(b) or a surface invariant by a family of parabolic isometries having the same fixed point at infinity,

(c) or a surface invariant by a family of hyperbolic isometries along the same fixed geodesic of $\mathbb{H}^{2}$.

Proof. Let $N$ be a unit normal field along $S$. We define the function $v$ on $S$ setting $v:=\left\langle N, \frac{\partial}{\partial t}\right\rangle$. We denote by $T$ the projection of $\frac{\partial}{\partial t}$ on $S$, that is $T=\frac{\partial}{\partial t}-v N$.

As the angle between $S$ and $\mathbb{M}^{2} \times\{t\}$ is constant along $S_{t}$, we deduce that $S_{t}$ is a line of curvature. Indeed let $\gamma: s \in I \subset \mathbb{R} \rightarrow \gamma(s) \in S_{t}$ be a regular parametrization of $S_{t}$, then taking into account that $\frac{\partial}{\partial t}$ is a parallel field and the definition of $T$,

$$
0=\frac{\mathrm{d}}{\mathrm{d} s}\left\langle N, \frac{\partial}{\partial t}\right\rangle=\left\langle\bar{\nabla}_{\gamma^{\prime}(s)} N, \frac{\partial}{\partial t}\right\rangle=\left\langle\bar{\nabla}_{\gamma^{\prime}(s)} N, T\right\rangle,
$$


where $\bar{\nabla}$ is the connection on $\mathbb{M}^{2} \times \mathbb{R}$. It follows that $T$ is a principal direction on $S$.

Let $c: u \in I \subset \mathbb{R} \rightarrow c(u) \in S$ be a line of curvature associated to the field $T$. We are going to show that $c(I)$ is contained in a vertical totally geodesic plane. This is equivalent to showing that the horizontal projection $c_{h}: I \subset \mathbb{R} \rightarrow \mathbb{M}^{2}$ of $c$ is a geodesic.

Assume first that $c$ is never vertical, that is $v \neq 0$ along $c$. Thus $c_{h}^{\prime}$ does not vanish.

Let $\nabla$ be the connection on $\mathbb{M}^{2}$. It is sufficient to show that $\nabla_{c_{h}^{\prime}} c_{h}^{\prime}$ is always parallel to $c_{h}^{\prime}$. As $c^{\prime}=c_{h}^{\prime}+\left(1-v^{2}\right) \frac{\partial}{\partial t}$ we have

$$
\begin{aligned}
\bar{\nabla}_{T} T & =\bar{\nabla}_{c^{\prime}} c^{\prime}=\nabla_{c_{h}^{\prime}} c_{h}^{\prime}+\frac{\mathrm{d}}{\mathrm{d} u}\left(1-v^{2}\right) \frac{\partial}{\partial t} \\
& =\nabla_{c_{h}^{\prime}} c_{h}^{\prime}-2 v v^{\prime} \frac{\partial}{\partial t} .
\end{aligned}
$$

As $T$ is a principal direction there exists a function $\lambda$ such that $\bar{\nabla}_{T} N=\lambda T$. Therefore

$$
v^{\prime}=\frac{\mathrm{d}}{\mathrm{d} u}\left\langle N, \frac{\partial}{\partial t}\right\rangle=\left\langle\bar{\nabla}_{T} N, \frac{\partial}{\partial t}\right\rangle=\lambda\left(1-v^{2}\right) .
$$

Thus we obtain

$$
\bar{\nabla}_{T} T=\nabla_{c_{h}^{\prime}} c_{h}^{\prime}-2 \lambda v\left(1-v^{2}\right) \frac{\partial}{\partial t}
$$

Moreover we have

$$
\begin{aligned}
\bar{\nabla}_{T} T & =\bar{\nabla}_{T}\left(\frac{\partial}{\partial t}-v N\right)=-\bar{\nabla}_{T} v N \\
& =-v^{\prime} N-\lambda v T \\
& =\left(\frac{v^{\prime}}{v}-\lambda v\right) c_{h}^{\prime}+\left(-v^{\prime} v-\lambda v\left(1-v^{2}\right)\right) \frac{\partial}{\partial t} \\
& =\left(\frac{v \prime}{v}-\lambda v\right) c_{h}^{\prime}-2 \lambda v\left(1-v^{2}\right) \frac{\partial}{\partial t} .
\end{aligned}
$$

Thus we get

$$
\nabla_{c_{h}^{\prime}} c_{h}^{\prime}=\left(\frac{\nu^{\prime}}{v}-\lambda v\right) c_{h}^{\prime}
$$

which shows that $c_{h}(I)$ is a geodesic in $\mathbb{M}^{2}$.

We denote by $w$ a unit horizontal field along $c$ tangent to $S$ and for each $u \in I$ we let $P(u)$ be the vertical totally geodesic plane containing $c(u)$ and orthogonal at $c(u)$ to $w(u)$.

Suppose now that $v$ vanishes on an open interval $J \subset I$. Let $u_{0} \in J$. Observe that along the horizontal curve of $S$ through $c\left(u_{0}\right)$ the vector field $N$ is horizontal. This means that an open set of $S$, including $c(J)$, is part of a cylinder $\gamma \times \mathbb{R}$ where 
$\gamma \subset \mathbb{M}^{2}$ is some horizontal curve. Clearly this implies that $w$ is constant along $J$, and thus so is $P$.

Combining those two arguments we see that $P$ is locally constant on an open and dense subset of $I$. As $P(u)$ depends in a differentiable way on $u$, we conclude that $P$ is constant.

Let us now consider a horizontal curve $\gamma: I \rightarrow S_{t}$ parametrized by arclength. Let $s_{1}, s_{2} \in I$ and call $\left.c:\right]-\varepsilon, \varepsilon\left[\rightarrow S\right.$ the integral curve of $T$ such that $c(0)=\gamma\left(s_{1}\right)$ and $\tilde{c}:]-\varepsilon, \varepsilon\left[\rightarrow S\right.$ the integral curve of $T$ such that $\tilde{c}(0)=\gamma\left(s_{2}\right)$.

Let us call $c_{3}$ (resp. $\tilde{c}_{3}$ ) the vertical coordinate of $c$ (resp. $\tilde{c}$ ). Calling again $u$ the parameter in $]-\varepsilon, \varepsilon[$, we have

$$
c_{3}^{\prime}(u)=\left\langle c^{\prime}(u), \frac{\partial}{\partial t}\right\rangle=\left\langle T(c(u)), \frac{\partial}{\partial t}\right\rangle=1-v^{2}(c(u))=1-v^{2}\left(c_{3}(u)\right) .
$$

Thus $c_{3}$ and $\tilde{c}_{3}$ verify the same first order differential equation with the same initial condition at $u=0$. We conclude that $c_{3} \equiv \tilde{c}_{3}$.

Recall that $c$ and $\tilde{c}$ are contained in vertical totally geodesic planes $P$ and $\widetilde{P}$.

Let us call $\Gamma \subset \mathbb{M}^{2}$ the complete constant geodesic curvature line defined by $\gamma$, that is $\gamma \subset \Gamma$.

Observe that there is a unique positive isometry $\varphi$ of $\mathbb{M}^{2}$ such that $\varphi(\Gamma)=\Gamma$, $\varphi(c(0))=\tilde{c}(0)$ and preserving the orientation of $\Gamma$. Therefore the isometry $\Phi(z, t)=$ $(\varphi(z), t)$ of $\mathbb{M}^{2} \times \mathbb{R}$ sends $P$ to $\widetilde{P}$. Note that the curves $\tilde{c}$ and $\Phi \circ c$ in the vertical plane $\widetilde{P}$ have the same vertical component and make the same angle with the horizontal for each $u \in]-\varepsilon, \varepsilon[$. We deduce that these curves coincide: $\Phi \circ c=\tilde{c}$. This concludes the proof.

We now state the main result of this section.

Theorem 16. Let $S \subset \mathbb{M}^{2} \times \mathbb{R}$ be an immersed totally umbilic surface. Then $S$ is part of a complete and embedded totally umbilic surface $\widetilde{S}$ which is invariant by a one-parameter group of isometries of $\mathbb{M}^{2} \times \mathbb{R}$. More precisely, up to an ambient isometry, in case $\mathbb{M}^{2}=\mathbb{S}^{2}$, then $\widetilde{S}$ is one of the examples described in Section 3, and in case $\mathbb{M}^{2}=\mathbb{H}^{2}$ then $\widetilde{S}$ is one of the examples described in Section 4.

In particular, any totally geodesic surface is part of a slice $\mathbb{M}^{2} \times \mathbb{R}$ or part of a product $\Gamma \times \mathbb{R}$ where $\Gamma \subset \mathbb{M}^{2}$ is a geodesic.

Proof. Locally $S$ is the image of an embedding $X: \Omega \rightarrow \mathbb{M}^{2} \times \mathbb{R}$, where $\Omega$ is an open disk in $\mathbb{R}^{2}$. As $X$ is totally umbilic, there exists a function $\lambda: \Omega \rightarrow \mathbb{R}$ such that

$$
\bar{\nabla}_{w} N=\lambda w
$$

for any vector $w$ tangent to $S$. 
Proceeding as in the proof of Theorem 1, as $\tau=0$ we obtain

$$
\nabla \lambda=\kappa \nu T,
$$

where $\kappa$ is the Gaussian curvature of $\mathbb{M}^{2}$, that is $\kappa=1$ if $\mathbb{M}^{2}=\mathbb{S}^{2}$ and $\kappa=-1$ if $\mathbb{M}^{2}=\mathbb{H}^{2}$.

Assume for the moment that $\lambda$ has no critical point. In particular each level curve of $\lambda$ is orthogonal to $T$ and is therefore horizontal, that is belongs to some $\mathbb{M}^{2} \times\left\{t_{0}\right\}$. Let $\gamma: I \subset \mathbb{M}^{2} \times\left\{t_{0}\right\}$ be such a curve parametrized by arclength. We have

$$
\frac{\mathrm{d} v}{\mathrm{~d} s}=\left\langle\bar{\nabla}_{\gamma^{\prime}(s)} N, \frac{\partial}{\partial t}\right\rangle+\left\langle N, \bar{\nabla}_{\gamma^{\prime}(s)} \frac{\partial}{\partial t}\right\rangle=\lambda(\gamma(s))\left\langle\gamma^{\prime}(s), \frac{\partial}{\partial t}\right\rangle=0,
$$

therefore $v$ is constant along $\gamma$.

We now call $n$ the unit normal field in $T\left(\mathbb{M}^{2} \times\left\{t_{0}\right\}\right)$ along $\gamma$ with the orientation induced by $N$. Let $\theta$ be the oriented angle between $n$ and $N$, hence $N(\gamma(s))=$ $\cos \theta(s) n(\gamma(s))+\sin \theta(s) \frac{\partial}{\partial t}(\gamma(s))$, we deduce that $\theta$ is constant along $\gamma$.

On the other hand,

$$
\begin{aligned}
\lambda(\gamma(s)) & =\left\langle\bar{\nabla}_{\gamma^{\prime}(s)} N, \gamma^{\prime}(s)\right\rangle \\
& =\left\langle\bar{\nabla}_{\gamma^{\prime}(s)}\left(\cos \theta n+\sin \theta \frac{\partial}{\partial t}\right), \gamma^{\prime}(s)\right\rangle \\
& =\cos \theta\left\langle\bar{\nabla}_{\gamma^{\prime}(s)} n, \gamma^{\prime}(s)\right\rangle+\sin \theta\left\langle\bar{\nabla}_{\gamma^{\prime}(s)} \frac{\partial}{\partial t}, \gamma^{\prime}(s)\right\rangle \\
& =\cos \theta\left\langle\bar{\nabla}_{\gamma^{\prime}(s)} n, \gamma^{\prime}(s)\right\rangle .
\end{aligned}
$$

Now observe that $\left\langle\bar{\nabla}_{\gamma^{\prime}(s)} n, \gamma^{\prime}(s)\right\rangle$ is the geodesic curvature of $\gamma$ in $\mathbb{M}^{2} \times\left\{t_{0}\right\}$. Since $\lambda$ and $\theta$ are constant along $\gamma$ we deduce that $\gamma$ has constant geodesic curvature. We conclude using Proposition 15 and results in Sections 3 and 4 that $S$ is as stated.

Suppose now that $\lambda$ has some critical points.

Let $U \subset S$ be a connected component, if any, of the interior of the set of critical points of $\lambda$. The formula (24) shows that $N$ is either always vertical or always horizontal in $U$. In the former case $U$ is part of a slice $\mathbb{M}^{2} \times\left\{t_{0}\right\}$ and in the latter case $U$ is part of a cylinder, that is part of a product $\Gamma \times \mathbb{R}$ where $\Gamma$ is some curve in $\mathbb{M}^{2}$. As $S$ is totally umbilic, $\Gamma$ has to be a geodesic and so $U$ is totally geodesic.

Let now $V \subset S$ be a connected component, if any, of the set of regular points of $\lambda$. From the first part of the proof, we know that $V$ is part of one of the symmetric examples given in Sections 3 and 4.

Therefore $S$ is obtained by gluing pieces of totally geodesic surfaces and pieces of the symmetric examples constructed in Sections 3 and 4. A closer look at these different types of surfaces shows that the whole of $S$ is either totally geodesic or part of one of the complete symmetric examples, which concludes the proof. 
Remark 17. The local existence of totally umbilic surfaces in $\mathbb{S}^{2} \times \mathbb{R}$ and $\mathbb{H}^{2} \times \mathbb{R}$ can be seen in an alternate way. Indeed, it is known that umbilicity is preserved by conformal diffeomorphisms, see [21], Volume 4 . It can be shown that $\mathbb{S}^{2} \times \mathbb{R}$ is conformally diffeomorphic to $\mathbb{R}^{3} \backslash\{(0,0,0)\}$, see Section 7. This implies the umbilic surfaces in $\mathbb{S}^{2} \times \mathbb{R}$ correspond through this conformal diffeomorphism to those of $\mathbb{R}^{3} \backslash\{(0,0,0)\}$. However to classify them in $\mathbb{S}^{2} \times \mathbb{R}$ up to congruences, and to understand their geometry requires a nontrivial work. Regarding $\mathbb{H}^{2} \times \mathbb{R}$, it can be shown that $\left.\mathbb{H}^{2} \times\right] 0, \pi$ [ is conformally diffeomorphic to $\mathbb{H}^{3}$, see Section 7 . Nonetheless this is not enough to understand the global geometry and topology of the umbilic surfaces in $\mathbb{H}^{2} \times \mathbb{R}$.

Remark 18. It can be proved that any twice differentiable totally umbilic surface in a space form, in $\mathbb{S}^{2}(\kappa) \times \mathbb{R}$ or in $\mathbb{H}^{2}(\kappa) \times \mathbb{R}$, is in fact $C^{3}$ and then analytic by the previous discussions, see [20].

\section{Totally umbilic surfaces in Sol}

The Sol geometry is the eighth model geometry of Thurston, see [22]. It is a Lie group endowed with a left-invariant metric, it is a homogeneous simply connected 3manifold with a 3-dimensional isometry group, see [3]. It is isometric to $\mathbb{R}^{3}$ equipped with the metric

$$
\mathrm{d} s^{2}=e^{2 z} \mathrm{~d} x^{2}+e^{-2 z} \mathrm{~d} y^{2}+\mathrm{d} z^{2} .
$$

The group structure of $\mathrm{Sol}$ is given by

$$
\left(x^{\prime}, y^{\prime}, z^{\prime}\right) \star(x, y, z)=\left(e^{-z^{\prime}} x+x^{\prime}, e^{z^{\prime}} y+y^{\prime}, z+z^{\prime}\right) .
$$

The isometries are

$$
(x, y, z) \mapsto\left( \pm e^{-c} x+a, \pm e^{c} y+b, z+c\right)
$$

and

$$
(x, y, z) \mapsto\left( \pm e^{-c} y+a, \pm e^{c} x+b,-z+c\right),
$$

where $a, b$ and $c$ are any real numbers. We set $E_{1}=e^{-z} \frac{\partial}{\partial x}, E_{2}=e^{z} \frac{\partial}{\partial y}$ and $E_{3}=\frac{\partial}{\partial z}$. Thus $\left(E_{1}, E_{2}, E_{3}\right)$ is a global orthonormal frame. A straightforward computation gives

$$
\begin{array}{lll}
\bar{\nabla}_{E_{1}} E_{1}=-E_{3}, & \bar{\nabla}_{E_{2}} E_{1}=0, & \bar{\nabla}_{E_{3}} E_{1}=0, \\
\bar{\nabla}_{E_{1}} E_{2}=0, & \bar{\nabla}_{E_{2}} E_{2}=E_{3}, & \bar{\nabla}_{E_{3}} E_{2}=0, \\
\bar{\nabla}_{E_{1}} E_{3}=E_{1}, & \bar{\nabla}_{E_{2}} E_{3}=-E_{2}, & \bar{\nabla}_{E_{3}} E_{3}=0 .
\end{array}
$$


We deduce from (25) that the vertical planes $\left\{x=x_{0}\right\}$ and $\left\{y=y_{0}\right\}$ are totally geodesic complete surfaces and that the horizontal planes $\left\{z=z_{0}\right\}$ are not totally umbilic surfaces (in fact they are minimal surfaces).

We now look for totally umbilic surfaces which are invariant under the one parameter group of isometries $(x, y, z) \mapsto(x+c, y, z)$. Clearly, such a surface is generated by a curve $\gamma$ in the totally geodesic plane $\{x=0\}$. Discarding the trivial case of a vertical plane $\left\{y=y_{0}\right\}$, we can assume that $\gamma$ locally is a graph over the $y$-axis. Thus $\gamma$ is given by $\gamma(y)=(0, y, z(y))$. Therefore the generated surface is parametrized by

$$
X(t, y):=(t, y, z(y)) .
$$

We have $X_{t}=(1,0,0)=e^{z} E_{1}$ and $X_{y}=\left(0,1, z^{\prime}\right)=e^{-z} E_{2}+z^{\prime} E_{3}$. As a unit normal field we can take

$$
N=\frac{e^{z} z^{\prime}}{\sqrt{1+e^{2 z} z^{\prime 2}}} E_{2}-\frac{1}{\sqrt{1+e^{2 z} z^{\prime 2}}} E_{3} .
$$

We have

$$
\begin{aligned}
\bar{\nabla}_{X_{t}} N= & -\frac{1}{\sqrt{1+e^{2 z} z^{\prime 2}}} X_{t} \\
\bar{\nabla}_{X_{y}} N= & \frac{e^{-z}}{\left(1+e^{2 z} z^{\prime 2}\right)^{3 / 2}}\left(1+2 e^{2 z} z^{\prime 2}+e^{2 z} z^{\prime \prime}\right) E_{2} \\
& \quad+\frac{z^{\prime}}{\left(1+e^{2 z} z^{\prime 2}\right)^{3 / 2}}\left(1+2 e^{2 z} z^{\prime 2}+e^{2 z} z^{\prime \prime}\right) E_{3}
\end{aligned}
$$

So that $X$ is a totally umbilic immersion if and only if

$$
\bar{\nabla}_{X_{y}} N=-\frac{1}{\sqrt{1+e^{2 z} z^{\prime 2}}} X_{y}
$$

that is, if and only if

$$
z^{\prime \prime}+3 z^{\prime 2}+2 e^{-2 z}=0
$$

A first integral of (26) is

$$
z^{\prime 2}=a e^{-6 z}-e^{-2 z},
$$

where $a$ is any positive real number.

Assume $z^{\prime}\left(y_{0}\right)=0$ for some $y_{0}$. Considering the function $f(y)=z\left(2 y_{0}-y\right)$, we can see that the curve $\gamma$ is symmetric with respect to the vertical geodesic $\left\{y=y_{0}\right\}$. Therefore, up to the isometry $(x, y, z) \mapsto(x,-y, z)$ and restricting the domain of $z$ if needed, we can assume $z^{\prime}>0$. Therefore $z$ satisfies

$$
z^{\prime}=e^{-z} \sqrt{a e^{-4 z}-1} .
$$


We consider the maximal solution of (27) defined by $z$ and we call it again $z$, it is defined on an open interval $] y_{1}, y_{2}\left[,-\infty \leq y_{1}<y_{2} \leq+\infty\right.$. By (27) the function $z$ is bounded above and is increasing we deduce using (26) that $z^{\prime \prime}$ has a negative limit at $y_{2}$. Taking into account the fact that $z^{\prime}$ is positive, we deduce that $y_{2}$ is finite, $y_{2}<+\infty$. Moreover, since $z^{\prime}$ is a positive and decreasing function, it has a nonnegative limit at $y_{2}$. If this limit were positive, we could extend the solution $z$ of (27) beyond $y_{2}$ which contradicts the maximality of $z$. Thus we have $\lim _{y \rightarrow y_{2}} z^{\prime}(y)=0$ and consequently $\lim _{y \rightarrow y_{2}} z(y)=\frac{1}{4} \log a$.

Consider now the maximal solution of (26) defined by $z$ and call it $z_{a}$. Since $z_{a}^{\prime}\left(y_{2}\right)=0$ we have $z_{a}\left(2 y_{2}-y\right)=z_{a}(y)$. Up to the horizontal translation $(x, y, z) \mapsto\left(x, y-y_{2}, z\right)$, which is an ambient isometry, we can assume that $y_{2}=0$ and therefore $z_{a}$ is an even function and is defined on an interval $]-y_{a}, y_{a}$ [ where $0<$ $y_{a} \leq+\infty$. Observe that there exist $A>0$ and $y_{3}>0$ such that $z_{a}^{\prime \prime}(y)<-A<0$ for any $y>y_{3}$. Therefore, if $y_{a}=+\infty$ we have $\lim _{y \rightarrow+\infty} z_{a}(y)=-\infty$. Suppose now that $y_{a}$ is finite. If $z_{a}$ had a finite limit at $y_{a}$ then $z_{a}^{\prime}$ would have also a finite limit but then we could extend the solution $z_{a}$ beyond $y_{a}$, which is absurd. We deduce that in both cases, that is $y_{a}<+\infty$ and $y_{a}=+\infty$, we have $\lim _{y \rightarrow y_{a}} z_{a}(y)=-\infty$.

Now we show that $y_{a}<+\infty$. Indeed, as $z_{a}$ is a solution of (26) satisfying $z_{a}(0)=0$ we have

$$
z^{\prime}(y)=-\sqrt{a} e^{-3 z} \sqrt{1-\frac{e^{4 z}}{a}},
$$

for any $y>0$. Since $\lim _{y \rightarrow y_{a}} z_{a}(y)=-\infty$, we deduce that for some $y_{4}>0$ we have

$$
\frac{1}{\sqrt{a}} z^{\prime} e^{3 z}<-\frac{1}{2}
$$

for any $y>y_{4}$. Therefore we have

$$
\frac{1}{3 \sqrt{a}} e^{3 z}<-\frac{y}{2}+c
$$

for some real constant $c$ and for any $y>y_{4}$. This implies that $y_{a}<+\infty$.

Call $\gamma_{a}$ the graph of the function $z_{a}, \gamma_{a}:=\left\{\left(0, y, z_{a}(y)\right),-y_{a}<y<y_{a}\right\}$ and call $F_{a}$ the totally umbilic complete surface generated by $\gamma_{a}$ :

$$
F_{a}:=\left\{\left(x, y, z_{a}(y)\right), x \in \mathbb{R},-y_{a}<y<y_{a}\right\} .
$$

Let $z_{a}$ and $z_{b}$ be two maximal solutions of (26) where $a$ and $b$ are any real numbers. Set $c=\frac{1}{4} \log \frac{b}{a}$ and consider the ambient isometry $(x, y, z) \mapsto\left(e^{-c} x, e^{c} y, z+c\right)$. Observe that this isometry maps the planar curve $\gamma_{a}$ onto the planar curve $\gamma_{b}$ and maps any Euclidean line parallel to the $x$-axis onto a line of the same type. Therefore this isometry maps the totally umbilic surface $F_{a}$ onto the totally umbilic surface $F_{b}$.

Summarizing, we state the following result. 
Proposition 19. Up to ambient isometries, there exist only two complete totally umbilic surfaces in the Sol group invariant under the horizontal translations $(x, y, z) \mapsto$ $(x+t, y, z), t \in \mathbb{R}$. The first one is the totally geodesic plane $\{y=0\}$. The second one is nongeodesic, is contained in a slab delimited by two totally geodesic planes $\left\{y= \pm y_{0}\right\}, y_{0}>0$, and is asymptotic to these planes. Moreover it is symmetric with respect to the totally geodesic plane $\{y=0\}$.

The generating curve in Sol for $a=\exp 8$

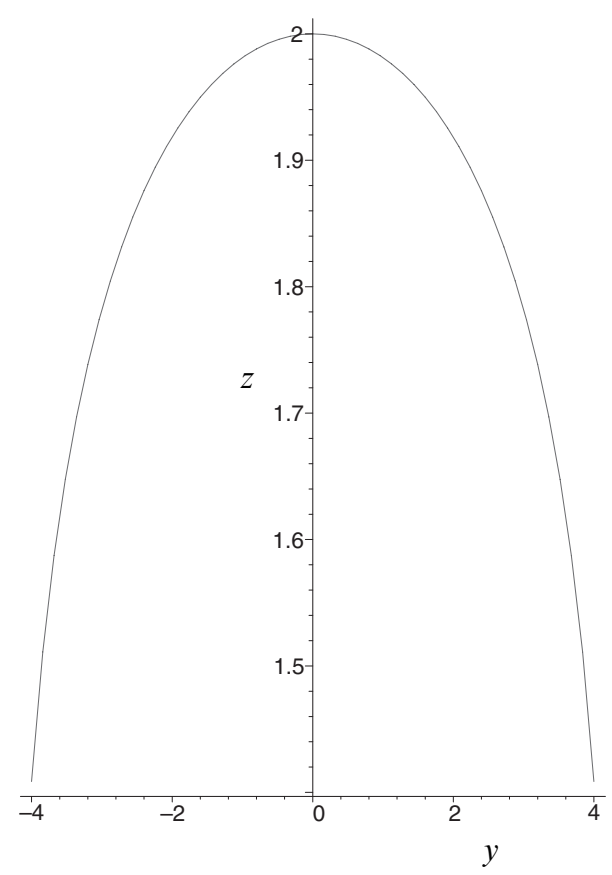

As a matter of fact we have the following.

Theorem 20. Up to ambient isometries, any totally umbilic surface in the Sol group is part of one of the two complete totally umbilic surfaces given in Proposition 19. In particular, up to ambient isometries, there exists a unique complete totally geodesic surface in the Sol group.

Proof. Let $S$ be an immersed totally umbilic surface in the Sol group. Locally $S$ is the image of an embedding $X: \Omega \rightarrow S o l$, where $\Omega$ is an open disk in $\mathbb{R}^{2}$. Call $(u, v)$ the coordinates on $\Omega$ and consider a unit normal field $N$ on $X(\Omega)$. As $X$ is totally 
umbilic, there exists a function $\lambda: \Omega \rightarrow \mathbb{R}$ such that

$$
\begin{aligned}
& \bar{\nabla}_{X_{u}} N=\lambda X_{u}, \\
& \bar{\nabla}_{X_{v}} N=\lambda X_{v},
\end{aligned}
$$

where $\bar{\nabla}$ is the Riemannian connection of the Sol group. As in the proof of Theorem 1 we find

$$
R\left(X_{u}, X_{v}\right) N=\lambda_{v} X_{u}-\lambda_{u} X_{v},
$$

where $R$ denotes the curvature tensor of the Sol group. Let us express the latter. Let $X, Y, Z$ and $W$ be any vector fields. Proceeding as in [7], Proposition 2.1, after some computations and using (25) we obtain the following:

$$
\begin{aligned}
\langle R(X, Y) Z, W\rangle=( & \langle X, Z\rangle\langle Y, W\rangle-\langle X, W\rangle\langle Y, Z\rangle) \\
& +2\left(\langle X, W\rangle\left\langle Y, \frac{\partial}{\partial z}\right\rangle\left\langle Z, \frac{\partial}{\partial z}\right\rangle+\langle Y, Z\rangle\left\langle X, \frac{\partial}{\partial z}\right\rangle\left\langle W, \frac{\partial}{\partial z}\right\rangle\right. \\
& \left.-\langle X, Z\rangle\left\langle Y, \frac{\partial}{\partial z}\right\rangle\left\langle W, \frac{\partial}{\partial z}\right\rangle-\langle Y, W\rangle\left\langle X, \frac{\partial}{\partial z}\right\rangle\left\langle Z, \frac{\partial}{\partial z}\right\rangle\right) .
\end{aligned}
$$

We define the function $v$ on $\Omega$ setting $v:=\left\langle N, \frac{\partial}{\partial z}\right\rangle$. We denote by $T$ the projection of $\frac{\partial}{\partial z}$ on $S$, that is $T=\frac{\partial}{\partial z}-v N$. We then have

$$
\begin{aligned}
R\left(X_{u}, X_{v}\right) N & =2 v\left(\left\langle X_{v}, \frac{\partial}{\partial z}\right\rangle X_{u}-\left\langle X_{u}, \frac{\partial}{\partial z}\right\rangle X_{v}\right) \\
& =2 v\left(\left\langle X_{v}, T\right\rangle X_{u}-\left\langle X_{u}, T\right\rangle X_{v}\right),
\end{aligned}
$$

wherefrom we deduce, using (28), that

$$
\nabla \lambda=2 \nu T .
$$

Assume first that $v$ and $T$ do not vanish on $\Omega$. Thus $T$ is of the form

$$
T=\alpha E_{1}+\beta E_{2}+\gamma E_{3},
$$

where $\alpha$ and $\beta$ do not vanish simultaneously. Since $|T|^{2}=1-v^{2}$ we have $\alpha^{2}+\beta^{2}=$ $v^{2}\left(1-v^{2}\right)$. We thus have

$$
N=-\frac{\alpha}{v} E_{1}-\frac{\beta}{v} E_{2}+v E_{3}
$$

We set

$$
J T=-\frac{\beta}{v} E_{1}+\frac{\alpha}{v} E_{2}
$$


so $J T$ is tangent to $S$, orthogonal to $T$ and $E_{3}$ and satisfies $|J T|^{2}=|T|^{2}$. Furthermore we have

$$
N \wedge T=J T, T \wedge J T=\left(1-v^{2}\right) N, J T \wedge N=T .
$$

We now compute the derivative $[T, J T](\lambda)$ in two different ways.

We first compute $[T, J T]=\bar{\nabla}_{T} J T-\bar{\nabla}_{J T} T$. We have

$$
\begin{aligned}
\bar{\nabla}_{T} J T & =\bar{\nabla}_{T} N \wedge T+N \wedge \bar{\nabla}_{T} T \\
& =N \wedge \bar{\nabla}_{T} T\left(\text { since } \bar{\nabla}_{T} N=\lambda N\right) \\
& =N \wedge \bar{\nabla}_{T}\left(E_{3}-v N\right) \\
& =N \wedge \bar{\nabla}_{T} E_{3}-\lambda \nu J T .
\end{aligned}
$$

Furthermore, using (25) we obtain

$$
\begin{aligned}
\bar{\nabla}_{T} E_{3} & =\alpha \bar{\nabla}_{E_{1}} E_{3}+\beta \bar{\nabla}_{E_{2}} E_{3}+\left(1-v^{2}\right) \bar{\nabla}_{E_{3}} E_{3} \\
& =\alpha E_{1}-\beta E_{2},
\end{aligned}
$$

wherefrom we deduce after some straightforward computations that

$$
\bar{\nabla}_{T} E_{3}=\frac{\alpha^{2}-\beta^{2}}{1-v^{2}} T-2 \frac{\alpha \beta}{v\left(1-v^{2}\right)} J T-v\left(1-v^{2}\right) N .
$$

Consequently

$$
\bar{\nabla}_{T} J T=2 \frac{\alpha \beta}{\nu\left(1-v^{2}\right)} T+\frac{\alpha^{2}-\beta^{2}}{1-v^{2}} J T-\lambda \nu J T .
$$

In the same way we obtain

$$
\begin{aligned}
\bar{\nabla}_{J T} T & =\bar{\nabla}_{J T} E_{3}-\bar{\nabla}_{J T} v N, \\
& =-2 \frac{\alpha \beta}{v\left(1-v^{2}\right)} T+v J T-\lambda v J T .
\end{aligned}
$$

We deduce that

$$
[T, J T]=4 \frac{\alpha \beta}{v\left(1-v^{2}\right)} T+\left(\frac{\alpha^{2}-\beta^{2}}{1-v^{2}}-v\right) J T .
$$

Using this last expression and (29), we find

$$
[T, J T](\lambda)=8 \alpha \beta .
$$


On the other hand, using again (29) we have

$$
\begin{aligned}
{[T, J T](\lambda) } & =T(J T(\lambda))-J T(T(\lambda)) \\
& =-J T(\langle\nabla \lambda, T\rangle) \\
& =2\left(-1+3 v^{2}\right) J T(v) \\
& =4 \frac{\alpha \beta}{v}\left(-1+3 v^{2}\right)
\end{aligned}
$$

since an easy computation gives $J T(v)=2 \alpha \beta / v$. From (30) and (31) we deduce

$$
\alpha \beta\left(3 v^{2}-2 v-1\right)=0 .
$$

Observe that if $v$ is constant on an open subset then $J T(v) \equiv 0$ which implies $\alpha \beta \equiv 0$. So in all cases we have $\alpha \beta \equiv 0$.

Recall that $\alpha$ and $\beta$ do not vanish simultaneously since by our assumption $v \neq 0$. Therefore we have either $\alpha \equiv 0$ or $\beta \equiv 0$.

Considering the isometry $(x, y, z) \mapsto(y, x,-z)$ we can assume that $\alpha \equiv 0$. Then the surface is part of a product $\mathbb{R} \times \Gamma$ where $\Gamma$ is a curve in the geodesic plane $\{x=0\}$. This case is considered in Proposition 19.

Let us suppose now that $T \equiv 0$ on an open set. Then this open set is part of a horizontal plane $\left\{z=z_{0}\right\}$, but this contradicts the assumption of umbilicity.

To finish the proof we consider the case where $v \equiv 0$ on an open subset. Therefore $T \equiv E_{3}$ and so this piece of the surface is part of a product $L \times \mathbb{R}$ where $L$ is a curve in the horizontal plane $\{z=0\}$. If $L$ is contained in a line parallel to the $y$-axis, then the surface is contained in a totally geodesic plane $\left\{x=x_{0}\right\}$. Discarding this trivial case, we can assume that $L$ is a graph over the $x$-axis. Consequently, the embedding $X$ is given by

$$
X(x, t)=(x, y(x), t)
$$

As a unit normal we take

$$
\begin{aligned}
N & =\frac{1}{\sqrt{e^{-2 t} y^{\prime 2}+e^{2 t}}}\left(e^{-2 t} y^{\prime},-e^{2 t}, 0\right) \\
& =\frac{y^{\prime}}{\sqrt{y^{\prime 2}+e^{4 t}}} E_{1}-\frac{1}{\sqrt{e^{-4 t} y^{\prime 2}+1}} E_{2} .
\end{aligned}
$$

As $X_{t}=E_{3}$, using (25) we obtain

$$
\bar{\nabla}_{X_{t}} N=-2 \frac{y^{\prime} e^{4 t}}{\left(y^{\prime 2}+e^{4 t}\right)^{3 / 2}} E_{1}-2 \frac{e^{-4 t} y^{\prime 2}}{\left(e^{-4 t} y^{\prime 2}+1\right)^{3 / 2}} E_{2} .
$$

The condition $\bar{\nabla}_{X_{t}} N=\lambda X_{t}$ is therefore equivalent to $\lambda \equiv 0$ and $y^{\prime} \equiv 0$. So $L$ is part of a line parallel to the $x$-axis and the surface is part of a geodesic plane $\left\{y=y_{0}\right\}$. This concludes the proof. 


\section{An application}

As an application of the classification of totally umbilic surfaces obtained in the previous sections, we can prove the following result:

Theorem 21. Any conformal diffeomorphism of $\mathbb{H}^{2} \times \mathbb{R}, \mathbb{S}^{2} \times \mathbb{R}$ and Sol is an isometry.

Proof. The result for $\mathbb{S}^{2} \times \mathbb{R}$ is a consequence of the fact that the mapping

$$
\begin{aligned}
\mathbb{S}^{2} \times \mathbb{R} & \rightarrow \mathbb{R}^{3}-\{(0,0,0)\}, \\
(p, t) & \mapsto e^{t} p
\end{aligned}
$$

is a conformal diffeomorphism -here $\mathbb{S}^{2}$ is viewed as the unit sphere of $\mathbb{R}^{3}$ centered at the origin. Indeed the conformal diffeomorphisms of $\mathbb{R}^{3}-\{(0,0,0)\}$ are the Möbius transformations fixing $(0,0,0)$ or sending $(0,0,0)$ to the point at infinity and these transformations correspond through the above conformal diffeomorphism to isometries of $\mathbb{S}^{2} \times \mathbb{R}$. We leave the details to the reader.

We now prove the result for the space $\mathbb{H}^{2} \times \mathbb{R}$. We claim that, except for the slices $\mathbb{H}^{2} \times\left\{t_{0}\right\}$, all the non-compact maximal (for the inclusion) totally umbilic surfaces in $\mathbb{H}^{2} \times \mathbb{R}$ are conformal to $\mathbb{C}$. This is clear for the products $\gamma \times \mathbb{R}$, where $\gamma \subset \mathbb{H}^{2}$ is a geodesic. As for the surfaces invariant under a one parameter group of parabolic transformations and which are all congruent to the surface $S_{P}$ described in 4.2, this is seen as follows. Consider in $\mathbb{H}^{3}$ a totally geodesic plane which we call $\mathbb{H}^{2}$ and denote by $N$ a unit normal along it. We let exp denote the exponential map in $\mathbb{H}^{3}$. Then the map

$$
\begin{aligned}
\left.\mathbb{H}^{2} \times\right] 0, \pi[ & \rightarrow \mathbb{H}^{3}, \\
(p, t) & \mapsto \exp _{p}\left(\ln \left(\tan \left(\frac{t}{2}\right)\right) N(p)\right)
\end{aligned}
$$

is a conformal diffeomorphism (cf. [20] for the details) which sends $S_{P}$ onto a totally umbilic surface of $\mathbb{H}^{3}$ with one point at infinity, that is a horosphere. It remains to consider the case of the surfaces invariant under a one parameter group of hyperbolic transformations. Consider such a surface $\Sigma$. We know that $\Sigma$ is invariant under a set of reflections of $\mathbb{H}^{2} \times \mathbb{R}$ through horizontal slices $\mathbb{H}^{2} \times\{t=a+n b\}$, for all $n \in \mathbb{Z}$, and $a, b \in \mathbb{R}$ depending on $\Sigma$. Suppose by contradiction that $\Sigma$ is conformal to $\mathbb{H}^{2}$. The isometries of $\Sigma$ induced by those reflections correspond then to conformal diffeomorphisms of $\mathbb{H}^{2}$ and so to isometries of $\mathbb{H}^{2}$. In particular all the horizontal curves $\Sigma \cap\{t=a n+b\}$ correspond to geodesics of $\mathbb{H}^{2}$. Now observe that all these curves are invariant by the hyperbolic isometries leaving $\Sigma$ invariant. We thus get isometries of $\mathbb{H}^{2}$ which leave (globally) invariant more than one geodesic. This is a contradiction as only the identity of $\mathbb{H}^{2}$ has this property. 
Take now a conformal diffeomorphism $\phi$ of $\mathbb{H}^{2} \times \mathbb{R}$. Then $\phi$ sends any horizontal slice $\mathbb{H}^{2} \times\left\{t_{0}\right\}$ to a maximal totally umbilic non-compact surface which is conformal to $\mathbb{H}^{2}$. From what precedes it follows that $\phi$ sends $\mathbb{H}^{2} \times\left\{t_{0}\right\}$ conformally to some horizontal slice $\mathbb{H}^{2} \times\left\{t_{1}\right\}$ and so isometrically. As $\phi$ is conformal this implies that for any $x \in \mathbb{H}^{2} \times\left\{t_{0}\right\}$, the tangent map $D_{x} \phi$ is an isometry. So $\phi$ is an isometry of $\mathbb{H}^{2} \times \mathbb{R}$.

The case of the Sol group is treated analogously. There are two maximal totally umbilic surfaces up to congruences. With the notations of Section 6, the first one is the totally geodesic plane $\left\{x=x_{0}\right\}$ and is easily seen to be isometric to $\mathbb{H}^{2}$. The second one is the surface parametrized by $X(t, y)=(t, y, z(y))$, where $t \in \mathbb{R}, y \in$ ] $-y_{1}, y_{1}$ [ and $z$ is the maximal solution to the equation $z^{\prime}=e^{-z} \sqrt{e^{-4 z}-1}$ (we have chosen $a=1$ with the notations of Section 6). The metric on this surface writes

$$
\mathrm{d} s^{2}=e^{2 z} \mathrm{~d} t^{2}+e^{-z} \mathrm{~d} y^{2} .
$$

Making the change of coordinate $\xi=\int e^{-4 z(y)} \mathrm{d} y$, the metric writes

$$
\mathrm{d} s^{2}=e^{2 z}\left(\mathrm{~d} t^{2}+\mathrm{d} \xi^{2}\right) .
$$

As the function $z$ is bounded from above (cf. Section 6), the flat metric $\mathrm{d} t^{2}+\mathrm{d} \xi^{2}$ is complete. It follows that the surface is conformal to $\mathbb{C}$. As the totally geodesic planes $\{x=$ const. $\}$ fill the whole space Sol, we conclude as before that any conformal diffeomorphism of $\mathrm{Sol}$ is an isometry.

\section{References}

[1] M. Abramowitz and A. Stegun, Handbook of mathematical functions. Dover Publications, Inc., New York, 1972. Zbl 0543.33001 MR

[2] U. Abresch and H. Rosenberg, A Hopf differential for constant mean curvature surfaces in $\mathbb{S}^{2} \times \mathbb{R}$ and $\mathbb{H}^{2} \times \mathbb{R}$. Acta Math. 193 (2004), 141-174. Zbl 1078.53053 MR 2134864

[3] F. Bonahon, Geometric structures on 3-manifolds. In Handbook of geometric topology, North-Holland, Amsterdam 2002, 93-164. Zbl 0997.57032 MR 1886669

[4] J. Aledo, J. Espinar and J. Gálvez, Complete surfaces of constant curvature in $\mathbb{H}^{2} \times \mathbb{R}$ and $\mathbb{S}^{2} \times \mathbb{R}$. Calc. Var. Partial Differential Equations 29 (2007), 347-363. Zbl 1119.53039 MR 2321892

[5] R. Caddeo, P. Piu and A. Ratto, SO(2)-invariant minimal and constant mean curvature surfaces in 3-dimensional homogeneous spaces. Manuscripta Math. 87 (1995), 1-12. Zbl 0827.53009 MR 1329436

[6] B. Daniel, Isometric immersions into $\mathbb{S}^{n} \times \mathbb{R}$ and $\mathbb{H}^{n} \times \mathbb{R}$ and applications to minimal surfaces. Preprint, 2004. arXiv:math/0406426v1

[7] B. Daniel, Isometric immersions into 3-dimensional homogeneous manifolds. Comment. Math. Helv. 82 (2007), 87-131. Zbl 1123.53029 MR 2296059 
[8] R. Sa Earp, Parabolic and hyperbolic screw motion surfaces in $\mathbb{H}^{2} \times \mathbb{R}$. J. Aust. Math. Soc. 85 (2008), 113-143. Zbl 05480757 MR 2460869

[9] R. Sa Earp and E. Toubiana, Screw motion surfaces in $\mathbb{H}^{2} \times \mathbb{R}$ and $\mathbb{S}^{2} \times \mathbb{R}$. Illinois J. Math. 49 (2005), 1323-1362. Zbl 1093.53068 MR 2210365

[10] I. Fernandez and P. Mira, Harmonic maps and constant mean curvature surfaces in $\mathbb{H}^{2} \times \mathbb{R}$. Amer. J. Math. 129 (2007), 1145-1181. Zbl 05186942 MR 2343386

[11] C. Figueroa, F. Mercuri and R. Pedrosa, Invariant surfaces of the Heisenberg groups. Ann Mat. Pura Appl. (IV) 177 (1999), 173-194. Zbl 0965.53042 MR 1747630

[12] L. Hauswirth, Minimal surfaces of Riemann type in three-dimensional product manifolds. Pacific J. Math. 224 (2006), 91-117. Zbl 1108.49031 MR 2231653

[13] L. Hauswirth, R. Sa Earp and E. Toubiana, Associate and conjugate minimal immersions in $\mathbb{M} \times \mathbb{R}$. Tohoku Math. J. (2) 60 (2008), 267-286. Zbl 1153.53041 MR 2428864

[14] W. Meeks III and H. Rosenberg, The theory of minimal surfaces in $M \times R$. Comment. Math. Helv. 80 (2005), 811-858. Zbl 1085.53049 MR 2182702

[15] B. Nelli and H. Rosenberg, Minimal surfaces in $H^{2} \times$ R. Bull. Braz. Math. Soc. 33 (2002), 263-292. Zbl 1038.53011 MR 2371953

[16] H. Rosenberg, Minimal surfaces in $\mathbb{M}^{2} \times \mathbb{R}$. Illinois J. Math. 46 (2002), 1177-1195. Zbl 1036.53008 MR 1988257

[17] A. Sanini, Gauss map of a surface of the Heisenberg group. Boll. Un. Mat. Ital. B (7) 11 (1997), 79-93. Zbl 0886.53018 MR 1456252

[18] G. Sansone and J. Gerretsen, Lectures on the theory of functions of a complex variable. P. Noordhoff, Groningen 1960. Zbl 0093.26803 MR 0113988

[19] P. Scott, The geometries of 3-manifolds. Bull. London Math. Soc. 15 (1983), 401-487. Zbl 0561.57001 MR 0705527

[20] R. Souam and E. Toubiana, On the classification and regularity of umbilic surfaces in homogeneous 3-manifolds. Mat. Contemp. 30 (2006), 201-215. Zbl 05356391 MR 2373511

[21] M. Spivak, A comprehensive introduction to differential geometry. Vol. 3 and 4, Publish or Perish, Boston, 1979. Zbl 0306.53001 Zbl 0306.53002 MR 0372756 MR 0394452

[22] W. Thurston, Three-dimensional geometry and topology. Princeton Math. Ser. 35, Princeton University Press, Princeton, NJ, 1997. Zbl 0873.57001 MR 1435975

Received October 1, 2007

Rabah Souam, Institut de Mathématiques de Jussieu, CNRS UMR 7586, Université Paris Diderot-Paris 7, Géométrie et Dynamique, Site Chevaleret, Case 7012, 75205 Paris Cedex 13, France

E-mail: souam@math.jussieu.fr

Eric Toubiana, Institut de Mathématiques de Jussieu, CNRS UMR 7586, Université Paris Diderot-Paris 7, Géométrie et Dynamique, Site Chevaleret, Case 7012, 75205 Paris Cedex 13, France

E-mail: toubiana@math.jussieu.fr 